JOURNAL OF INTEGRAL EQUATIONS

AND APPLICATIONS

Volume 6, Number 1, Winter 1994

\title{
THE JACOBI METHOD IN WEIGHTED BANACH SPACES FOR INTEGRAL EQUATIONS, WITH EMPHASIS ON GREEN'S- FUNCTION-LIKE KERNELS
}

\author{
L. KOHAUPT
}

\begin{abstract}
The theoretical foundations for the iterative solution of integral equations in weighted function spaces are derived which allow a unified treatment of the following two cases. Case 1: the kernel is nonzero on its whole domain of definition. Case 2: the kernel is nonzero in the interior of its domain of definition and zero or partly zero on the boundary, which often occurs with Green's functions. The method presented is new, particularly the theory of condensing operators according to [10, pp. 102-109] can be avoided, which simplifies the treatment of the corresponding integral equations considerably. Further, strong and weak convergence criteria are given. The results are used to sharpen error estimates in the Jacobi method (i.e., in the method of successive approximations) and are applied to a boundary value problem. Numerical tests show good agreement with the theoretical results.
\end{abstract}

0. Introduction. There are important relationships between the dynamic analysis of elastic structures in engineering science and the iterative methods of positive completely continuous operators in numerical mathematics. In dynamics, eigenfrequencies and eigenfunctions play a fundamental role. For many problems the first eigenfrequency and corresponding eigenfunction are most important. Often, it is not sufficient to know merely the first eigenfrequency. Also, for iteration methods for positive completely continuous operators, the greatest eigenvalue (i.e., the spectral radius) and the corresponding positive eigenfunction are of similar importance.

The significance of the spectral radius is widely known: Under appropriate conditions, an iteration process for general operator equations converges, if the spectral radius of the iteration operator is less than one. It is less known (or at least, it is less made use of) that the (positive) eigenfunction corresponding to the spectral radius of an integral

Received by the editors on April 5, 1993.

Copyright (C)1994 Rocky Mountain Mathematics Consortium 
operator can be used to sharpen error estimates. Namely, by means of weighted norms one succeeds in establishing optimal estimates, since the sup-norm of the iteration operator equals the spectral radius if the corresponding eigenfunction is taken as a weight function.

The spectral properties (more precisely the greatest eigenvalue and corresponding eigenvector) of positive operators are investigated thoroughly in [10, Chapter 1], but the (positive) eigenvector corresponding to the spectral radius isn't used so as to sharpen estimates. This may be demonstrated by the following example. In case of an integral operator whose kernel is positive throughout its domain of definition, under appropriate conditions the best rate of convergence is obtained in the weighted norm $\max _{x \in \Omega}|u(x)| / \kappa(x)$ where $\kappa(x)$ is the positive eigenfunction corresponding to the spectral radius. But when the kernel equals zero on the boundary of the domain of definition, the eigenfunction also equals zero on the boundary $\partial \Omega$ of $\Omega$ so that the norm $\max _{x \in \Omega}|u(x)| / \kappa(x)$ is now undefined. In this case in [10, pp. 102-109] the theory of condensing operators is applied, which is rather complicated even for simple Green's functions. For instance, in the example given, only $B^{2}$ is condensing, not $B$ itself. It seems that a unified treatment of both cases has not yet been developed.

Therefore, the main objective of this paper consists in treating both cases for linear Fredholm integral equations in a unified manner. When the operator $B$ with kernel $K(x, s)$ is not positive, the operator $|B|$ with kernel $|K(x, s)|$ is positive, and the corresponding eigenfunction can be used to sharpen error estimates. In Section 2 it is shown that the real weighted function spaces $C_{\sigma^{-1}}(\Omega)$ are complete, and that the considered integral operators in these spaces are completely continuous. Further, the eigenvalues are estimated, an inclusion for the spectral radius as well as strong and weak convergence criteria are stated.

In Section 3 the corresponding statements for the real weighted function spaces $L_{\sigma}(\Omega)$ are obtained. In Section 4 specific examples illustrate the general theorems of Sections 2 and 3. Finally, as an application of the general results, in Section 5 the numerical solution of a boundary value problem (problem of a loaded elastic string) is carried out by means of the Jacobi method. For this, the ordinary differential equation describing the problem is cast into a linear Fredholm equation of the second kind. 
1. Preliminaries and notation. For some theorems in a Banach space $V$, e.g., in the spectral theory of completely continuous operators $B$, the space $V$ has to be complex. In case of real spaces (which is assumed in the next sections) the corresponding theorems are valid for the complexifications $\tilde{V}$ and $\tilde{B}$ of $V$ and $B$. As to the complexifications, cf., e.g., [11, Section 4] and [8, especially Section 2]. In real Banach spaces, for instance, $\Sigma\left(B^{*}\right)=\Sigma(B)$ holds where $\Sigma(B)$ is the spectrum of $B$ (cf. [11, Section 4]). When the space is real, the adjoint operator $B^{*}$ is also denoted by $B^{T}$ so that $\Sigma\left(B^{T}\right)=\Sigma(B)$. In the following sections, $B^{T}$ denotes an integral operator with the adjoint kernel of $B$, where $B^{T}$ is not always the adjoint operator of $B$. Nevertheless, $\Sigma^{\prime}\left(B^{T}\right)=\Sigma^{\prime}(B)$ with $\Sigma^{\prime}(B):=\Sigma(B) \backslash\{0\}$ will remain valid. For a Banach space $V$, by $\mathcal{B}(V)$ the Banach space of continuous operators in $V$ is denoted. If $B \in \mathcal{B}(V)$ is completely continuous, we write $B: V \hookrightarrow V$. By $\Omega=[a, b]$, we denote a bounded closed interval of the real line, $C(\Omega)$ is the space of continuous functions on $\Omega$ with norm $\|u\|_{\infty}=\max _{x \in \Omega}|u(x)|, u \in C(\Omega), L(\Omega)$ is the space of integrable functions on $\Omega$ with norm $\|u\|_{1}=\int_{\Omega}|u(x)| d x, u \in L(\Omega)$, and $L_{2}(\Omega)$ is the space of measurable square summable functions on $\Omega$ with norm $\|u\|_{2}=\left(\int_{\Omega}|u(x)|^{2} d x\right)^{1 / 2}, u \in L_{2}(\Omega)$. Let $\stackrel{\circ}{\Omega}=\operatorname{int}(\Omega)$. By $H^{1}(\stackrel{\circ}{\Omega})$ we denote the Sobolev space of functions on $\stackrel{\circ}{\Omega}$ which are, along with their first derivative in the sense of distributions, elements of $L_{2}(\stackrel{\circ}{\Omega})$; the norm in $H^{1}(\stackrel{\circ}{\Omega})$ is defined by $\|u\|=\left(\|u\|_{2}^{2}+\left\|u^{\prime}\right\|_{2}^{2}\right)^{1 / 2}, u \in H^{1}(\stackrel{\circ}{\Omega})$. Further, $H_{0}^{1}(\stackrel{\circ}{\Omega})$ means the space of functions $u \in H^{1}(\stackrel{\circ}{\Omega})$ with the property $\left.u\right|_{\partial \Omega}=0$, where $\partial \Omega=\{a, b\}$ is the boundary of $\Omega$.

In order to prove the existence of positive eigenvectors $\kappa$, respectively $\chi$, corresponding to the spectral radius $\rho(B)$, respectively $\rho\left(B^{*}\right)=$ $\rho(B)$, one can use $[\mathbf{1 1}$, Theorem 6.1 , respectively Theorem 6.2] or [10, Theorem 9.9]. In case of the adjoint integral operator $B^{*}=B^{T}$, one has to first consider the mapping $B^{T}: L_{2}(\Omega) \hookrightarrow L_{2}(\Omega)$; then one uses $B^{T}: L_{2}(\Omega) \rightarrow C(\Omega)$.

In the Jacobi method for the solution of the matrix equation $u-B u=f$ with positive matrix $B$, error estimates can be derived using the spectral radius of the iteration matrix instead of a norm of $B$ provided an appropriate weighted norm is chosen. For the application of the row-sum criterion, e.g., one needs the norm 
$\|u\|_{\infty, \sigma^{-1}}:=\max _{i=1, \ldots, n}\left|u_{i}\right| / \sigma_{i}, u=\left(u_{1}, \ldots, u_{n}\right) \in \mathbf{K}^{n}$ with a weight vector $\sigma=\left(\sigma_{1}, \ldots, \sigma_{n}\right)>0$; likewise, for the application of the columnsum criterion, the norm $\|u\|_{1, \sigma}:=\sum_{i=1}^{n} \sigma_{i}\left|u_{i}\right|, u \in \mathbf{K}^{n}$ is needed.

For linear Fredholm integral equations of the second kind, one correspondingly applies the weighted norm

$$
\|u\|_{\infty, \sigma^{-1}}:=\max _{x \in \Omega} \frac{|u(x)|}{\sigma(x)}, \quad u \in C(\Omega),
$$

respectively

$$
\|u\|_{1, \sigma}:=\int_{\Omega} \sigma(x)|u(x)| d x, \quad u \in L(\Omega),
$$

where it is assumed for the moment that $\sigma \in C(\Omega), \sigma(x)>0$, $x \in \Omega$. Choosing as $\sigma$ the eigenfunction $\kappa$ corresponding to the greatest eigenvalue $\rho_{\infty}(|B|)$ of $(|B| u)(x)=\int_{\Omega}|K(x, s)| u(s) d s, x \in \Omega$, $u \in C(\Omega)$, respectively choosing as $\sigma$ the eigenfunction $\chi$ corresponding to the greatest eigenvalue $\rho_{\infty}\left(|B|^{T}\right) \quad\left(=\rho_{\infty}(|B|)\right)$ of the operator $\left(|B|^{T} u\right)(x)=\int_{\Omega}|K(s, x)| u(s) d s, x \in \Omega, u \in C(\Omega)$, the norm $\|\cdot\|_{\infty, \sigma^{-1}}$ has to be redefined if $\sigma(x)=0, x \in N=N_{\sigma} \subset \partial \Omega$, e.g., for the integral operator with kernel

$$
K(x, s)=G(x, s)= \begin{cases}s, & 0 \leq s \leq x \leq l \\ x, & 0 \leq x \leq s \leq l\end{cases}
$$

(i.e., $K(x, s)=\min \{s, x\})$ one has according to [5], respectively [7], the relations

$$
\rho_{\infty}(|B|)=\rho_{\infty}(B)=\rho_{\infty}\left(B^{T}\right)=\rho_{1}(B)=\rho_{1}\left(B^{T}\right)=\left(\frac{2 l}{\pi}\right)^{2}
$$

and

$$
\kappa(x)=\chi(x)=\sin \left(\frac{\pi}{2 l} x\right), \quad x \in \Omega:=[0, l],
$$

where $N=\{0\} \subset \partial \Omega=\{0, l\}$.

For the operator $B$ whose Green's-function kernel is

$$
K(x, s)=G(x, s)= \begin{cases}\left(1-\frac{x}{l}\right) s, & 0 \leq s \leq x \leq l, \\ \left(1-\frac{s}{l}\right) x, & 0 \leq x \leq s \leq l,\end{cases}
$$


one has the relations

$$
\rho_{\infty}(|B|)=\rho_{\infty}(B)=\rho_{\infty}\left(B^{T}\right)=\rho_{1}(B)=\rho_{1}\left(B^{T}\right)=\left(\frac{l}{\pi}\right)^{2}
$$

and

$$
\kappa(x)=\chi(x)=\sin \left(\frac{\pi}{l} x\right), \quad x \in \Omega=[0, l],
$$

where $N=\{0, l\}=\partial \Omega$.

It should be noted that the operator $|B|^{T}: C(\Omega) \rightarrow C(\Omega)$ is not the adjoint operator of $|B|: C(\Omega) \rightarrow C(\Omega)$. Finally, it is remarked that the error estimates in Section 5 are based on the contraction-mapping theorem.

2. Theory in space $C_{\sigma^{-1}}(\Omega)$. In this section the space $C_{\sigma^{-1}}(\Omega)$ is defined which is a generalization of the space $C(\Omega)$. It can be shown that $C_{\sigma^{-1}}(\Omega)$ is complete. Further, it is proven that the integral operator $B$ in $C_{\sigma^{-1}}(\Omega)$ is completely continuous, extensions of the integral kernel $K_{\sigma^{-1}}(\cdot, \cdot)$ from $\Omega_{N} \times \Omega_{N}$ to $\Omega \times \Omega$ are considered, an estimate of the eigenvalues as well as an inclusion of the spectral radius are derived, and convergence criteria are stated.

2.1. The space $C_{\sigma^{-1}}(\Omega)$. Let $\Omega=[a, b]$ and $\sigma \in C(\Omega)$. Further, let $N=N_{\sigma} \subset \partial \Omega, \Omega_{N}=\Omega_{N_{\sigma}}:=\Omega \backslash N$ and $\sigma(x)>0, x \in \Omega_{N}, \sigma(x)=0$, $x \in N=N_{\sigma}$. Then the set

$$
\begin{aligned}
C_{\sigma^{-1}}(\Omega): & =\left\{u \in C(\Omega) \mid \lim _{\substack{y \rightarrow x \\
y \in \Omega_{N}}} \frac{u(y)}{\sigma(y)} \text { exists for all } x \in N\right\} \\
& =\left\{u \in C(\Omega) \mid \exists q \in C(\Omega) \text { with } q(x)=\frac{u(x)}{\sigma(x)}, x \in \Omega_{N}\right\}
\end{aligned}
$$

forms a vector space with the property $u(x)=0, x \in N$. A norm is defined on $C_{\sigma^{-1}}(\Omega)$ by

$$
\|u\|_{\infty, \sigma^{-1}}:=\sup _{x \in \Omega_{N}} \frac{|u(x)|}{\sigma(x)}=\sup _{x \in \Omega_{N}}|q(x)|=\|q\|_{\infty} .
$$


In case $N=N_{\sigma}=\varnothing$, one has $C_{\sigma^{-1}}(\Omega)=C(\Omega)$, and the norms $\|\cdot\|_{\infty, \sigma^{-1}}$ and $\|\cdot\|_{\infty}$ are equivalent; for $N \neq \varnothing$, one has $C_{\sigma^{-1}}(\Omega) \subset C(\Omega)$ and $\|u\|_{\infty} \leq \sigma_{\max }\|u\|_{\infty, \sigma^{-1}}, u \in C_{\sigma^{-1}}(\Omega)$, i.e., the embedding is continuous, and convergence in the norm $\|\cdot\|_{\infty, \sigma^{-1}}$ implies convergence in the norm $\|\cdot\|_{\infty}$.

Theorem 2.1.1. The space $C_{\sigma^{-1}}(\Omega)$ in (1) endowed with the norm (2) is complete, i.e., $C_{\sigma^{-1}}(\Omega)$ is a Banach space.

Proof. Let $\left(u_{n}\right)_{n \in \mathbf{N}}$ be a Cauchy sequence in $C_{\sigma^{-1}}(\Omega)$, and let $q_{n}$ be the continuous extension of $u_{n}(x) / \sigma(x), x \in \Omega_{N}$, to the entire interval $\Omega$ for $n \in \mathbf{N}$. Let $\varepsilon>0$. Then there exists a number $n_{0} \in \mathbf{N}$ such that

$$
\left\|q_{n}-q_{m}\right\|_{\infty}=\left\|u_{n}-u_{m}\right\|_{\infty, \sigma^{-1}} \leq \varepsilon \quad \text { for all } n, m \geq n_{0} .
$$

Therefore, $\left(q_{n}\right)_{n \in \mathbf{N}}$ is a Cauchy sequence in $C(\Omega)$. Since $C(\Omega)$ is complete, there exists a function $q \in C(\Omega)$ with

$$
\left\|q_{n}-q\right\|_{\infty} \rightarrow 0, \quad n \rightarrow \infty .
$$

Let

$$
u(x):=q(x) \sigma(x), \quad x \in \Omega .
$$

Then $u \in C_{\sigma^{-1}}(\Omega)$, and one has

$$
\left\|u_{n}-u\right\|_{\infty, \sigma^{-1}}=\left\|q_{n}-q\right\|_{\infty} \leq \varepsilon, \quad n \geq n_{0} .
$$

Hence, $C_{\sigma^{-1}}(\Omega)$ is complete.

2.2. Complete continuity of integral operators in $C_{\sigma^{-1}}(\Omega)$. The next question is under what conditions the integral operator

$$
(B u)(x)=\int_{\Omega} K(x, s) u(s) d s, \quad x \in \Omega
$$

with kernel

$$
K(\cdot, \cdot) \in C(\Omega \times \Omega)
$$


defines a bounded mapping in $C_{\sigma^{-1}}(\Omega)$. After this, it is investigated when this operator is completely continuous. Sometimes, the kernel has to satisfy stronger conditions. For this, let $N=N_{K} \subset \partial \Omega$, $\Omega=\Omega_{N_{K}}=\Omega \backslash N_{K}$ and

$$
\begin{aligned}
& K(\cdot, \cdot) \in C(\Omega \times \Omega) \\
& K(x, s) \neq 0, \quad x, s \in \Omega_{N} \\
& K(x, s)=0 \quad \begin{cases}x \in N, & s \in \Omega_{N} \\
s \in N, & x \in \Omega_{N} \\
x \in N, & s \in N .\end{cases}
\end{aligned}
$$

Remark 1. Usually one has $N_{K}=N_{\sigma}$; but also the case $N_{\sigma}=\varnothing \subset$ $N_{K}$ occurs, e.g., when $\sigma(x) \equiv 1$. The condition (3) is assumed when $\sigma=\kappa$, respectively $\sigma=\chi$, is the eigenfunction of $|B|: C(\Omega) \rightarrow C(\Omega)$, respectively, of $|B|^{T}: C(\Omega) \rightarrow C(\Omega)$ corresponding to the spectral radius $\rho_{\infty}(|B|)$; then $\sigma(x)>0$ for $x \in \Omega_{N}$ and $\sigma(x)=0$ for $x \in N$. Under condition (3), according to [10, Theorem 9.9, p. 95] there exists a nonnegative eigenfunction $\kappa$, respectively $\chi$, of $|B|$, respectively $|B|^{T}$, corresponding to the eigenvalue $\rho_{\infty}(|B|)=\rho_{\infty}\left(|B|^{T}\right)$. Kernels having property (3) are denoted as Green's-function-like kernels. The symmetry of the kernels is not assumed even though Green's functions are symmetric, as a rule. Besides, condition (3) is needed for the weak convergence criteria in Section 2.5.

Remark 2. Under condition (3), the operator in (1) is reducible according to [10, p. 119] if $N \neq \varnothing$. But choosing $V=C_{N}(\Omega):=\{u \in$ $C(\Omega) \mid u(x)=0, x \in N\}$ with $N=N_{K}$, the operator $B$ is irreducible according to $[\mathbf{1 0}$, p. 110].

Subsequently, we shall see in Section 4, Example 3, that the kernel $K(\cdot, \cdot)$ may also have a finite number of logarithmic discontinuities. Generally, the kernel must be such that $B: C(\Omega) \hookrightarrow C(\Omega)$, its eigenfunction $\kappa$, respectively, $\chi$, has the property $\kappa(x)>0, x \in \Omega_{N}$, $\kappa(x)=0, x \in N$, respectively $\chi(x)>0, x \in \Omega_{N}, \chi(x)=0, x \in N$, and $B$ is irreducible in an appropriate subspace of $C(\Omega)$. This is also required in case of the space $L_{\sigma}(\Omega)$.

Necessary and sufficient conditions on the kernel to guarantee all these properties seem to be unknown. 
After these remarks, we continue the investigation of the integral operator. The operator (1) with kernel (2) can be written in the form

$$
(B u)(x)=\int_{\Omega} \frac{K(x, s) \sigma(s)}{\sigma(x)} \sigma(x) \frac{u(s)}{\sigma(s)} d s .
$$

Let $q(x)$ be the continuous extension of $u(x) / \sigma(x), x \in \Omega_{N}$, to $\Omega$, and

$$
K_{\sigma^{-1}}(s, x):=\frac{K(x, s) \sigma(s)}{\sigma(x)}, \quad x, s \in \Omega_{N} .
$$

Further, let there exist a function $\hat{K}_{\sigma^{-1}}(\cdot, \cdot)$ on $\Omega \times \Omega$ with the property

$$
\begin{aligned}
\hat{K}_{\sigma^{-1}}(\cdot, \cdot) & \in C(\Omega \times \Omega) \\
\hat{K}_{\sigma^{-1}}(x, s) & =K_{\sigma^{-1}}(x, s), \quad x, s \in \Omega_{N},
\end{aligned}
$$

i.e., it is assumed that $K_{\sigma^{-1}}(\cdot, \cdot) \in C\left(\Omega_{N} \times \Omega_{N}\right)$ possesses a continuous extension to $\Omega \times \Omega$ (which is then uniquely determined). In case $N_{\sigma}=\varnothing$, condition (6) is trivially satisfied so that (6) is only of interest for $\varnothing \neq N_{\sigma}=N_{K}$. Under condition (6), it follows that

$$
(B u)(x)=\int_{\Omega} \hat{K}_{\sigma^{-1}}(x, s) \sigma(x) q(s) d s, \quad x \in \Omega, q(x)=\frac{u(x)}{\sigma(x)}, \quad x \in \Omega_{N},
$$

$u \in C_{\sigma^{-1}}(\Omega)$, whence

$$
B: C_{\sigma^{-1}}(\Omega) \rightarrow C_{\sigma^{-1}}(\Omega) .
$$

Then the following holds.

Theorem 2.2.1. Assume (2) and (6). Then the operator

$$
B: C_{\sigma^{-1}}(\Omega) \rightarrow C_{\sigma^{-1}}(\Omega)
$$

is completely continuous.

Proof. Let $\left(u_{n}\right)_{n \in \mathbf{N}}$ be a bounded sequence in $C_{\sigma^{-1}}(\Omega)$, and let $q_{n}$ be a continuous extension of $u_{n}(x) / \sigma(x), x \in \Omega_{N}$, to $\Omega$ for $n \in \mathbf{N}$. Then there exists a constant $\gamma>0$ satisfying

$$
\left\|q_{n}\right\|_{\infty}=\left\|u_{n}\right\|_{\infty, \sigma^{-1}} \leq \gamma, \quad n \in \mathbf{N}
$$


Let

$$
\left(\hat{B}_{\sigma^{-1}} u\right)(x):=\int_{\Omega} \hat{K}_{\sigma^{-1}}(x, s) q(s) d s, \quad x \in \Omega,
$$

$u \in C_{\sigma^{-1}}(\Omega)$, where $q$ denotes the continuous extension of $u / \sigma$ to $\Omega$. Because of

$$
\hat{B}_{\sigma^{-1}}: C_{\sigma^{-1}}(\Omega) \hookrightarrow C(\Omega)
$$

and (9), there is a subsequence $\mathbf{N}^{\prime} \subset \mathbf{N}$ and an element $w \in C(\Omega)$ with the property

$$
\left\|\hat{B}_{\sigma^{-1}} u_{n}-w\right\|_{\infty} \rightarrow 0, \quad n \in \mathbf{N}^{\prime} .
$$

Let

$$
v(x):=w(x) \sigma(x), \quad x \in \Omega .
$$

Then one has

$$
\left\|B u_{n}-v\right\|_{\infty, \sigma^{-1}}=\left\|\hat{B}_{\sigma^{-1}} u_{n}-w\right\|_{\infty} \rightarrow 0, \quad n \in \mathbf{N}^{\prime} .
$$

As $v \in C_{\sigma^{-1}}(\Omega)$, the proof is complete.

Remark 3. The norm of the operator (8) satisfies

$$
\begin{aligned}
\|B\|_{\infty, \sigma^{-1}}: & =\sup _{0 \neq u \in C_{\sigma^{-1}}(\Omega)} \frac{\|B u\|_{\infty, \sigma^{-1}}}{\|u\|_{\infty, \sigma^{-1}}}=\max _{x \in \Omega} \int_{\Omega}\left|\hat{K}_{\sigma^{-1}}(x, s)\right| d s \\
& =\sup _{x \in \Omega_{N}} \int_{\Omega}\left|K_{\sigma^{-1}}(x, s)\right| d s \\
& =\sup _{x \in \Omega_{N}} \frac{1}{\sigma(x)} \int_{\Omega}|K(x, s)| \sigma(s) d s .
\end{aligned}
$$

Remark 4. If, besides (3), condition (6) is also satisfied for $\sigma=\kappa$, then

$$
\rho_{\infty}(|B|)=\|B\|_{\infty, \kappa^{-1}}=\sup _{x \in \Omega_{N}} \frac{1}{\kappa(x)} \int_{\Omega}|K(x, s)| \kappa(s) d s .
$$


2.3. Extension of the integral kernel $K_{\sigma^{-1}}(\cdot, \cdot)$. In this subsection, for a weight function $\sigma$, sufficient conditions are specified ensuring that the integral kernel $K_{\sigma^{-1}}(\cdot, \cdot) \in C\left(\Omega_{N} \times \Omega_{N}\right)$ has an extension $\hat{K}_{\sigma^{-1}}(\cdot, \cdot) \in C(\Omega \times \Omega)$. For this, we consider the set $P_{*, \kappa}$, where $\kappa$ is the nonnegative eigenfunction corresponding to the spectral radius $\rho\left(|B|: C(\Omega) \rightarrow C(\Omega)\right.$ ). Then $P_{*, \kappa}$ contains the functions $\sigma$ allowing an extension of $K_{\sigma^{-1}}(\cdot, \cdot)$.

Let condition 2.2 (3) be satisfied. From [10, Theorem 9.9] or [11, Theorem 6.1], one infers that the integral operator

$$
|B|: C(\Omega) \rightarrow C(\Omega)
$$

has an eigenfunction $\sigma=\kappa$ such that $\kappa(x)>0, x \in N=N_{K}$ and $\kappa(x)=0, x \in N=N_{k}$ where $\kappa$ is the eigenfunction corresponding to the spectral radius $\rho_{\infty}(|B|):=\rho(|B|: C(\Omega) \rightarrow C(\Omega))>0$, which is also an eigenvalue of $(1)$. It is supposed that the extension $\hat{K}_{\kappa^{-1}}(\cdot, \cdot)$ of $K_{\kappa^{-1}}(\cdot, \cdot)$ exists, i.e., it is assumed that

$$
\begin{aligned}
\hat{K}_{\kappa^{-1}}(\cdot, \cdot) & \in C(\Omega \times \Omega) \\
\hat{K}_{\kappa^{-1}}(x, 1) & =K_{\kappa^{-1}}(x, s), \quad x, s \in \Omega_{N} .
\end{aligned}
$$

Under condition (2), let

$$
\begin{aligned}
& P_{*, \kappa}:=\left\{\sigma \in C(\Omega) \mid \sigma(x)>0, x \in \Omega_{N} ; \sigma(x)=0, x \in N ;\right. \\
& \text { the extensions } \hat{q}_{\sigma^{-1}} \in C(\Omega) \text { of } q_{\sigma^{-1}}(x):=\frac{\kappa(x)}{\sigma(x)}, x \in \Omega_{N}, \\
& \text { and } \left.\hat{q}_{\sigma} \in C(\Omega) \text { of } q_{\sigma}(x):=\frac{\sigma(x)}{\kappa(x)}, x \in \Omega_{N}, \text { exist }\right\} .
\end{aligned}
$$

As $\kappa \in P_{*, \kappa}$, one has $P_{*, \kappa} \neq \varnothing$. Then the following theorem holds.

Theorem 2.3.1. Let the conditions 2.2(3) and (2) be satisfied; further, let $\sigma$ be a function from $P_{*, \kappa}$. Then the kernel $K_{\sigma^{-1}}(\cdot, \cdot) \in$ $C\left(\Omega_{N} \times \Omega_{N}\right)$ has an extension $\hat{K}_{\sigma^{-1}}(\cdot, \cdot) \in C(\Omega \times \Omega)$, i.e.,

$$
\begin{aligned}
\hat{K}_{\sigma^{-1}}(\cdot, \cdot) & \in C(\Omega \times \Omega) \\
\hat{K}_{\sigma^{-1}}(x, s) & =K_{\sigma^{-1}}(x, s), \quad x, s \in \Omega_{N}
\end{aligned}
$$

for all $\sigma \in P_{*, \kappa}$. 
The proof is simple and therefore omitted. Further, the following theorem holds.

Theorem 2.3.1. Assume the conditions 2.2(3) and (2). Then

$$
C_{\sigma^{-1}}(\Omega)=C_{\kappa^{-1}}(\Omega), \quad \sigma \in P_{*, \kappa}
$$

as well as

(6)

$$
\gamma_{0}\|u\|_{\infty, \sigma^{-1}} \leq\|u\|_{\infty, \kappa^{-1}} \leq \gamma_{1}\|u\|_{\infty, \sigma^{-1}}, \quad u \in C_{\sigma^{-1}}(\Omega)=C_{\kappa^{-1}}(\Omega)
$$

with

(7) $\gamma_{0}=\frac{1}{\sup _{x \in \Omega_{N}} \frac{\kappa(x)}{\sigma(x)}}=\frac{1}{\left\|\hat{q}_{\sigma^{-1}}\right\|_{\infty}}, \quad \gamma_{1}=\sup _{x \in \Omega_{N}} \frac{\sigma(x)}{\kappa(x)}=\left\|\hat{q}_{\sigma}\right\|_{\infty}$.

Thus, $C_{\sigma^{-1}}(\Omega)$ and $C_{\kappa^{-1}}(\Omega)$ are equal considered as vector spaces, and the associated norms are equivalent.

Proof. (i) Let $u \in C_{\sigma^{-1}}(\Omega)$. This entails $\|u\|_{\infty, \sigma^{-1}}<\infty$ and hence

$$
\sup _{x \in \Omega_{N}} \frac{|u(x)|}{\kappa(x)} \leq \sup _{x \in \Omega_{N}} \frac{|u(x)|}{\sigma(x)} \sup _{x \in \Omega_{N}} \frac{\sigma(x)}{\kappa(x)}=\gamma_{1}\|u\|_{\infty, \sigma^{-1}}
$$

with $\gamma_{1}=\sup _{x \in \Omega_{N}} \sigma(x) / \kappa(x)<\infty$. Therefore $u \in C_{\kappa^{-1}}(\Omega)$, and consequently the right-hand part in (6) is proven.

(ii) Let $u \in C_{\kappa^{-1}}(\Omega)$. Then $\|u\|_{\infty, \kappa^{-1}}<\infty$ and therefore

$$
\sup _{x \in \Omega_{N}} \frac{|u(x)|}{\sigma(x)} \leq \sup _{x \in \Omega_{N}} \frac{|u(x)|}{\kappa(x)} \sup _{x \in \Omega_{N}} \frac{\kappa(x)}{\sigma(x)}=\frac{1}{\gamma_{0}}\|u\|_{\infty, \kappa^{-1}}
$$

with $\gamma_{0}=1 / \sup _{x \in \Omega_{N}} \kappa(x) / \sigma(x)$. Hence $u \in C_{\sigma^{-1}}(\Omega)$, and consequently the left-hand part in (6) is shown.

2.4. Estimation of the eigenvalues and inclusion of the spectral radius for $C_{\sigma^{-1}}(\Omega)$. The results of this subsection are used in 2.5 in order to establish strong and weak convergence criteria. Instead of assuming the integral operator to be irreducible in case of the weak criteria, condition 2.2(3) is considered. One has 
Theorem 2.4.1. Let the conditions $2.2(3)$ and 2.2(6) be satisfied; further, let $\nu_{\sigma^{-1}}>0$ be any number and

$$
\int_{\Omega}\left|\hat{K}_{\sigma^{-1}}(x, s)\right| d s \leq \nu_{\sigma^{-1}}, \quad x \in \Omega
$$

as well as

$$
\int_{\Omega}\left|\hat{K}_{\sigma^{-1}}\left(x_{0}, s\right)\right| d s<\nu_{\sigma^{-1}} \quad \text { for at least one } x_{0} \in \Omega \text {. }
$$

Then,

(3) $|\lambda|<\nu_{\sigma^{-1}}$, for all $\lambda \in \Sigma_{\infty, \sigma^{-1}}:=\Sigma\left(B: C_{\sigma^{-1}}(\Omega) \hookrightarrow C_{\sigma^{-1}}(\Omega)\right)$.

Remark 1. In case $N_{K} \neq \varnothing$ and $\sigma \equiv 1$, i.e., $N_{\sigma}=\varnothing$, condition (2) is satisfied for all $x_{0} \in N_{K} \subset \partial \Omega$.

Proof of Theorem 1. (i) $\sigma \equiv 1$.

Assumption 1. Let

$$
|\lambda| \geq \nu_{\sigma^{-1}} \quad \text { for a } \lambda \in \Sigma_{\infty, \sigma^{-1}} .
$$

Then

$$
|\lambda| \geq \int_{\Omega}|K(x, s)| d s, \quad x \in \Omega
$$

and

$$
|\lambda|>\int_{\Omega}\left|K\left(x_{0}, s\right)\right| d s
$$

Assumption 2. Let

$$
\left|w\left(x_{0}\right)\right|=\left|w\left(x_{m}\right):=\max _{x \in \Omega}\right| w(x) \mid
$$


where $w$ is an eigenfunction corresponding to $\lambda$. Then one has

$$
\left|w\left(x_{m}\right)\right|>0
$$

and hence

$$
\begin{aligned}
|\lambda| \mid w\left(x_{0} \mid\right. & >\int_{\Omega}\left|K\left(x_{0}, s\right)\right| d s\left|w\left(x_{0}\right)\right| \\
& =\int_{\Omega}\left|K\left(x_{0}, s\right)\right|\left|w\left(x_{m}\right)\right| d s \\
& \geq \int_{\Omega}\left|K\left(x_{0}, s\right)\right||w(s)| d s .
\end{aligned}
$$

On the other hand, one has

$$
|\lambda||w(x)| \leq \int_{\Omega}|K(x, s)||w(s)| d s, \quad x \in \Omega,
$$

i.e., (9) contradicts (10). If Assumption 1 were false, there would be nothing to prove. So, Assumption 2 must be false. Therefore,

$$
\left|w\left(x_{0}\right)\right|<\left|w\left(x_{m}\right)\right|
$$

and, consequently,

$$
|w(x)|<\left|w\left(x_{m}\right)\right|, \quad x \in S=S\left(x_{0}\right)
$$

for an open ball $S\left(x_{0}\right)$ with center $x_{0}$. Let

$$
T:=\Omega \backslash S .
$$

Taking into account (4), one has

$$
\begin{aligned}
|\lambda|\left|w\left(x_{m}\right)\right| & \geq \int_{\Omega}\left|K\left(x_{m}, s\right)\right|\left|w\left(x_{m}\right)\right| d s \\
& >\int_{S}\left|K\left(x_{m}, s\right)\right||w(s)| d s+\int_{T}\left|K\left(x_{m}, s\right)\right||w(s)| d s \\
& =\int_{\Omega}\left|K\left(x_{m}, s\right)\right||w(s)| d s .
\end{aligned}
$$


This contradicts (10). Therefore, Assumption 1 is also false, and hence relation (3) must be true.

(ii) General case. Let

$$
\left(\hat{B}_{\sigma^{-1}} u\right)(x):=\int_{\Omega} \hat{K}_{\sigma^{-1}}(x, s) u(s) d s, \quad x \in \Omega,
$$

$u \in C(\Omega)$. Then

$$
\hat{B}_{\sigma^{-1}}: C(\Omega) \hookrightarrow C(\Omega)
$$

and one has

$$
\hat{\Sigma}_{\infty, \sigma^{-1}}^{\prime}:=\Sigma^{\prime}\left(\hat{B}_{\sigma^{-1}}: C(\Omega) \hookrightarrow C(\Omega)\right)=\Sigma_{\infty, \sigma^{-1}}^{\prime} .
$$

From this and (i), the assertion follows.

For the proof of the next theorem we need

Lemma 1. Let the condition $2.2(3)$ be satisfied, and additionally let $K(x, s) \geq 0, x, s \in \Omega$. Then either

$$
\int_{\Omega} K(x, s) d s=\rho_{\infty}(B), \quad x \in \Omega
$$

or

$$
\min _{x \in \Omega} \int_{\Omega} K(x, s) d s<\rho_{\infty}(B)<\max _{x \in \Omega} \int_{\Omega} K(x, s) d s
$$

Proof. Let Equation (18) be false. Subsequently, only the left hand part of (19) is proven. In case $N \neq \varnothing$, one has $0=\int_{\Omega} K\left(x_{0}, s\right) d s$ for an $x_{0} \in N$. Further, $\rho_{\infty}(B)>0$ so that the assertion follows. In case $N=\varnothing$, i.e., $K(x, s)>0, x, s \in \Omega$, according to [10, Lemma 9.1] the relation $\rho_{\infty}(B) \geq \alpha:=\min _{x \in \Omega} \int_{\Omega} K(x, s) d s$ holds.

Now it is assumed that $\min _{x \in \Omega} \int_{\Omega} K(x, s) d s=\rho_{\infty}(B)$. Let $x_{i} \in$ $\Omega$ be a point with $\int_{\Omega} K\left(x_{i}, s\right) d s=\min _{x \in \Omega} \int_{\Omega} K(x, s) d s$. Then $\int_{\Omega} K\left(x_{i}, s\right) d s=\rho_{\infty}(B)$. When (18) is false (as assumed), one 
has $\int_{\Omega} K\left(x_{i}, s\right) d s<\int_{\Omega} K(x, s) d s, x \in S\left(x_{a}\right)$, where $S\left(x_{a}\right)$ is a ball with center $x_{a}$ and where $x_{a}$ is such that $\int_{\Omega} K\left(x_{a}, s\right) d s=$ $\max _{x \in \Omega} \int_{\Omega} K(x, s) d s$. Let $\kappa$ be a positive eigenfunction corresponding to the eigenvalue $\rho_{\infty}(B)$. Then

$$
\rho_{\infty}(B)\left[\kappa(x)-\kappa\left(x_{i}\right)\right]=\int_{\Omega}\left[K(x, s)-K\left(x_{i}, s\right)\right] \kappa(s) d s, \quad x \in S\left(x_{a}\right),
$$

and therefore

$$
\kappa(x)>\kappa\left(x_{i}\right)>0, \quad x \in S\left(x_{a}\right),
$$

i.e.,

$$
\frac{\kappa(s)}{\kappa\left(x_{i}\right)}>1, \quad s \in S\left(x_{a}\right)
$$

Hence,

$$
\rho_{\infty}(B)=\int_{\Omega} \frac{K\left(x_{i}, s\right) \kappa(s)}{\kappa\left(x_{i}\right)} d s>\int_{\Omega} K\left(x_{i}, s\right) d s,
$$

which is a contradiction to $\rho_{\infty}(B)=\int_{\Omega} K\left(x_{i}, s\right) d s$. Therefore, the above assumption must be false. Consequently, there exists a point $x_{0} \in \Omega$ with the property $\int_{\Omega} K\left(x_{0}, s\right) d s<\rho_{\infty}(B)$. The proof of the right-hand inequality is similar to that of the left-hand one.

Theorem 2. Assume 2.2(3) and $K(x, s) \geq 0, x, s \in \Omega$. Additionally let 2.3(2) be satisfied. Then, for all $\sigma \in P_{*, \kappa}$ either

$$
\int_{\Omega} \hat{K}_{\sigma^{-1}}(x, s) d s=\rho_{\infty}(B), \quad x \in \Omega
$$

or

$$
\min _{x \in \Omega} \int_{\Omega} \hat{K}_{\sigma^{-1}}(x, s) d s<\rho_{\infty}(B)<\max _{x \in \Omega} \int_{\Omega} \hat{K}_{\sigma^{-1}}(x, s) d s .
$$

Furthermore,

$$
\begin{aligned}
\sup _{\sigma \in P_{*, \kappa}} \min _{x \in \Omega} \int_{\Omega} \hat{K}_{\sigma^{-1}}(x, s) d s & \\
=\rho_{\infty}(B) & =\inf _{\sigma \in P_{*, \kappa}} \max _{x \in \Omega} \int_{\Omega} \hat{K}_{\sigma^{-1}}(x, s) d s .
\end{aligned}
$$


Proof. Let $\hat{B}_{\sigma^{-1}}: C(\Omega) \hookrightarrow C(\Omega)$ be defined by

$$
\left(\hat{B}_{\sigma^{-1}} u\right)(x):=\int_{\Omega} \hat{K}_{\sigma^{-1}}(x, s) u(s) d s, \quad x \in \Omega .
$$

According to Lemma 1, either

$$
\int_{\Omega} \hat{K}_{\sigma^{-1}}(x, s) d s=\rho_{\infty}\left(\hat{B}_{\sigma^{-1}}\right), \quad x \in \Omega
$$

or

$$
\text { (29) } \min _{x \in \Omega} \int_{\Omega} \hat{K}_{\sigma^{-1}}(x, s) d s<\rho_{\infty}\left(\hat{B}_{\sigma^{-1}}\right)<\max _{x \in \Omega} \int_{\Omega} \hat{K}_{\sigma^{-1}}(x, s) d s .
$$

Now

$$
\int_{\Omega} K(x, s) \kappa(s) d s=\rho_{\infty}(B) \kappa(x), \quad x \in \Omega
$$

whence

$$
\rho_{\infty}(B)=\int_{\Omega} \hat{K}_{\kappa^{-1}}(x, s) d s, \quad x \in \Omega
$$

This implies

$$
\begin{aligned}
\rho_{\infty}(B) & =\rho_{\infty}\left(\hat{B}_{\kappa^{-1}}\right)=\rho_{\infty, \kappa^{-1}}(B) \\
& =\rho\left(B: C_{\kappa^{-1}}(\Omega) \hookrightarrow C_{\kappa^{-1}}(\Omega)\right) \\
& =\rho_{\infty, \sigma^{-1}}(B)=\rho_{\infty}\left(\hat{B}_{\sigma^{-1}}\right), \quad \sigma \in P_{*, \kappa},
\end{aligned}
$$

so that (24) and (25) are proven. Moreover, from (24) and (25) one infers

$$
\begin{aligned}
\text { (33) } \sup _{\sigma \in P_{*, \kappa}} \min _{x \in \Omega} \int_{\Omega} \hat{K}_{\sigma^{-1}}(x, s) d s & \\
\leq & \leq \rho_{\infty}(B) \leq \inf _{\sigma \in P_{*, \kappa}} \max _{x \in \Omega} \int_{\Omega} \hat{K}_{\sigma^{-1}}(x, s) d s .
\end{aligned}
$$


The equality sign follows, in each case, because on the left hand side, respectively right hand side, the supremum, respectively infimum, is taken on for $\sigma=\kappa$. Hence, Theorem 2 is proven.

Finally we remark that

$$
\rho_{\infty, \sigma^{-1}}(B) \leq \rho_{\infty, \sigma^{-1}}(|B|) .
$$

2.5. The convergence criteria in $C_{\sigma^{-1}}(\Omega)$. As a first application of the estimates for the eigenvalues, strong and weak convergence criteria are derived; they correspond to the strong and weak row-sum criteria for matrices. In case of the strong criteria, it is assumed that $K(\cdot, \cdot) \in C(\Omega \times \Omega)$, and in case of the weak criteria, condition 2.2(3) is supposed additionally, which will not be mentioned subsequently.

(i) Let $N=N_{\sigma}=\phi$ and $\sigma \equiv 1$, i.e., $C_{\sigma^{-1}}(\Omega)=C(\Omega)$ and $\|\cdot\|_{\infty, \sigma^{-1}}=\|\cdot\|_{\infty} ;$ let

$$
q:=\max _{x \in \Omega} \int_{\Omega}|K(x, s)| d s<1 .
$$

Then we say that the strong "row-integral criterion" (for short: the strong RIC) is fulfilled. In this case $\|B\|_{\infty}=q<1$ holds. For the weak RIC, let

$$
\begin{gathered}
\int_{\Omega}|K(x, s)| d s \leq 1, \quad x \in \Omega \\
\int_{\Omega}\left|K\left(x_{0}, s\right)\right| d s<1 \quad \text { for at least one } x_{0} \in \Omega, \text { if } N_{K}=\varnothing .
\end{gathered}
$$

Then, according to subsection 2.4, the relation $\rho_{\infty}(|B|)<1$ holds.

(ii) Let additionally the condition 2.2(6) be satisfied and

$$
q:=\max _{x \in \Omega} \int_{\Omega}\left|\hat{K}_{\sigma^{-1}}(x, s)\right| d s<1 .
$$

Then we say that the strong $\sigma^{-1}$-RIC is fulfilled. In this case $\|B\|_{\infty, \sigma^{-1}}=q<1$. 
For the weak $\sigma^{-1}$-RIC, let

$$
\begin{gathered}
\int_{\Omega}\left|\hat{K}_{\sigma^{-1}}(x, s)\right| d s \leq 1, \quad x \in \Omega \\
\int_{\Omega}\left|\hat{K}_{\sigma^{-1}}\left(x_{0}, s\right)\right| d s<1 \quad \text { for at least one } x_{0} \in \Omega .
\end{gathered}
$$

Then $\rho_{\infty, \sigma^{-1}}(|B|)<1$.

(iii) Let additionally condition 2.3(2) be satisfied and

$$
\int_{\Omega}\left|\hat{K}_{\kappa^{-1}}\left(x_{0}, s\right)\right| d s<1 \quad \text { for at least one } x_{0} \in \Omega \text {. }
$$

Condition (3) is called $\kappa^{-1}$-RIC; here the strong and weak criterion are equivalent. Under this condition, $\rho_{\infty, \kappa^{-1}}(|B|)=\|B\|_{\infty, \kappa^{-1}}<1$.

3. Theory in space $L_{\sigma}(\Omega)$. In this section the space $L_{\sigma}(\Omega)$ is defined, which is a generalization of the space $L(\Omega)$. For $L_{\sigma}(\Omega)$, theorems corresponding to the case $C_{\sigma^{-1}}(\Omega)$ are proven.

\subsection{The space $L_{\sigma}(\Omega)$. Let}

$$
L_{\sigma}(\Omega):=\left\{u \mid u \text { is measurable on } \Omega, \text { and } \int_{\Omega}|u(x)| \sigma(x) d x<\infty\right\} .
$$

Then $L_{\sigma}(\Omega)$ is a vector space. A norm is defined on $L_{\sigma}(\Omega)$ by

$$
\|u\|_{1, \sigma}:=\int_{\Omega}|u(x)| \sigma(x) d x, \quad u \in L_{\sigma}(\Omega) .
$$

In case $N=\varnothing$, one has $L_{\sigma}(\Omega)=L(\Omega)$, and the norms $\|\cdot\|_{1, \sigma}$ and $\|\cdot\|_{1}$ are equivalent. In case $N \neq \varnothing$ one only has $L(\Omega) \subset L_{\sigma}(\Omega)$ and $\|u\|_{1, \sigma} \leq \sigma_{\max }\|u\|_{1}, u \in L(\Omega)$, i.e., the embedding is continuous, and the convergence in the norm $\|\cdot\|_{1}$ entails the convergence in the norm $\|\cdot\|_{1, \sigma}$. One has

Theorem 3.1.1. The space $L_{\sigma}(\Omega)$ endowed with the norm (2) is complete, i.e., $L_{\sigma}(\Omega)$ is a Banach space. 
Proof. Let $\left(u_{n}\right)_{n \in \mathbf{N}}$ be a Cauchy sequence with $u_{n} \in L_{\sigma}(\Omega), n \in \mathbf{N}$. Then $u_{n}$ is measurable, and $\left\|u_{n}\right\|_{1, \sigma}<\infty, n \in \mathbf{N}$, holds. Further, let $\varepsilon>0$ be arbitrarily chosen, but fixed. Then there exists a number $n_{0} \in \mathbf{N}$ such that

$$
\int_{\Omega} \sigma(x)\left|u_{n}(x)-u_{m}(x)\right| d x \leq \varepsilon, \quad n, m \geq n_{0} .
$$

The functions $\sigma u_{n}$ are measurable for $n \in \mathbf{N}$, and because of (3), $\left(\sigma u_{n}\right)_{n \in \mathbf{N}}$ is a Cauchy sequence in $L(\Omega)$. As $L(\Omega)$ is complete, there is an element $v \in L(\Omega)$ such that

$$
\int_{\Omega}\left|\sigma(x) u_{m}(x)-v(x)\right| d x \rightarrow 0, \quad m \rightarrow \infty
$$

holds. Hence, one also has

$$
\sigma u_{m} \stackrel{\text { meas }}{\rightarrow} v, \quad m \rightarrow \infty .
$$

Fatou's theorem (cf. $\left[\mathbf{1 3}\right.$, p. 155]) applied to the sequence $\sigma(x) \mid u_{m}(x)-$ $u_{n}(x)|=| \sigma(x) u_{m}(x)-\sigma(x) u_{n}(x) \mid$ along with (3)-(5) yields the relation

$$
\begin{aligned}
\int_{\Omega} \mid \sigma(x) & u_{n}(x)-v(x) \mid d x \\
& \leq \sup _{m \geq n} \int_{\Omega}\left|\sigma(x) u_{n}(x)-\sigma(x) u_{m}(x)\right| d x \leq \varepsilon, \quad n, m \geq n_{0},
\end{aligned}
$$

Let

$$
u(x):=\frac{u(x)}{\sigma(x)}, \quad x \in \Omega_{N} .
$$

Then $u$ is measurable (cf. [13, Section 1 and IV, Section 2]). Consequently, one has

(8) $\int_{\Omega} \sigma(x)\left|u_{n}(x)-u(x)\right| d x=\int_{\Omega}\left|\sigma(x) u_{n}(x)-v(x)\right| d x \leq \varepsilon, \quad n \geq n_{0}$.

Hence,

$$
u \in L_{\sigma}(\Omega)
$$


and

$$
u_{n} \rightarrow u, \quad n \rightarrow \infty \quad \text { in } L_{\sigma}(\Omega)
$$

Altogether, it has been shown that $L_{\sigma}(\Omega)$ is complete.

3.2. Complete continuity of integral operators in $L_{\sigma}(\Omega)$. The operator 2.2(1) with kernel $2.2(2)$ can be cast into the form

$$
(B u)(x) \sigma(x)=\int_{\Omega} \frac{\sigma(x) K(x, s)}{\sigma(s)} u(s) \sigma(s) d s .
$$

Let

$$
K_{\sigma}(x, s):=\frac{\sigma(x) K(x, s)}{\sigma(s)}, \quad x, s \in \Omega_{N},
$$

and let there exist a function $\hat{K}_{\sigma}(\cdot, \cdot)$ on $\Omega \times \Omega$ such that

$$
\begin{aligned}
\hat{K}_{\sigma}(\cdot, \cdot) & \in C(\Omega \times \Omega) \\
\hat{K}_{\sigma}(x, s) & =K_{\sigma}(x, s), \quad x, s \in \Omega_{N},
\end{aligned}
$$

i.e., it is assumed that there exists a continuous extension of $K_{\sigma}(\cdot, \cdot) \in$ $C\left(\Omega_{N} \times \Omega_{N}\right)$ to $\Omega \times \Omega$ (which is then uniquely determined). Hence (1) has the representation

$$
(B u)(x) \sigma(x)=\int_{\Omega} \hat{K}_{\sigma}(x, s) u(s) \sigma(s) d s, \quad x \in \Omega,
$$

$u \in L_{\sigma}(\Omega)$. From this, one concludes that

$$
\sigma B: L_{\sigma}(\Omega) \hookrightarrow C(\Omega)
$$

holds, where $\sigma B$ is defined by $(\sigma B u)(x)=\sigma(x)(B u)(x), x \in \Omega$. Consequently, the following theorem holds.

Theorem 3.2.1. Let the condition 2.2(2) and the condition (3) be fulfilled. Then the operator

$$
B: L_{\sigma}(\Omega) \rightarrow L_{\sigma}(\Omega)
$$

is completely continuous. 
Remark 1 . The norm of the operator (6) is given by (7)

$$
\begin{aligned}
\|B\|_{1, \sigma}: & =\sup _{0 \neq u \in L_{\sigma}(\Omega)} \frac{\|B u\|_{1, \sigma}}{\|u\|_{1, \sigma}}=\max _{s \in \Omega} \int_{\Omega}\left|\hat{K}_{\sigma}(x, s)\right| d x \\
& =\sup _{s \in \Omega_{N}} \int_{\Omega}\left|K_{\sigma}(x, s)\right| d x=\sup _{s \in \Omega_{N}} \frac{1}{\sigma(s)} \int_{\Omega} \sigma(x)|K(x, s)| d x .
\end{aligned}
$$

Remark 2. If $K(\cdot, \cdot)$ satisfies condition $2.2(3)$, then the operator $|B|^{T}: C(\Omega) \hookrightarrow C(\Omega)$ has eigenvalues different from zero. Hence, the conditions of [11, Theorem 6.1] are fulfilled. Consequently, $\rho_{\infty}^{T}:=$ $\rho\left(|B|^{T}\right)$ is an eigenvalue of $|B|^{T}$ and has an eigenfunction $\chi \in C(\Omega)$, $\chi(x) \geq 0, x \in \Omega$. Because of 2.2(3), it follows immediately that $\chi(x)>0, x \in \Omega_{N}$, and $\chi(x)=0, x \in N$. If condition (3) is also fulfilled for $\sigma=\chi$, then

$$
\rho_{\infty}\left(|B|^{T}\right)=\|B\|_{1, \chi}=\sup _{s \in \Omega_{N}} \frac{1}{\chi(s)} \int_{\Omega} \chi(x)|K(x, s)| d x .
$$

3.3. Extension of the integral kernel $K_{\sigma}(\cdot, \cdot)$. Let the condition 2.2(3) be satisfied. According to 3.2, Remark 2, the operator

$$
|B|^{T}: C(\Omega) \hookrightarrow C(\Omega)
$$

has an eigenfunction $\sigma=\chi \in C(\Omega)$ with $\chi(x)>0, x \in \Omega_{N_{K}}$, and $\chi(x)=0, x \in N=N_{K}$, corresponding to the eigenvalue

$$
\begin{aligned}
\rho_{\infty}^{T}: & =\rho\left(|B|^{T}: C(\Omega) \hookrightarrow C(\Omega)\right) \\
& =\rho_{\infty}:=\rho(|B|: C(\Omega) \hookrightarrow C(\Omega)) \\
& =\rho_{1}:=\rho(|B|: L(\Omega) \hookrightarrow L(\Omega)) \\
& =\rho_{1}^{T}:=\rho\left(|B|^{T}: L(\Omega) \hookrightarrow(\Omega)\right)>0 .
\end{aligned}
$$

We suppose that the extension $\hat{K}_{\chi}(\cdot, \cdot)$ of $K_{\chi}(\cdot, \cdot)$ exists, i.e., that

$$
\begin{aligned}
\hat{K}_{\chi}(\cdot, \cdot) & \in C(\Omega \times \Omega) \\
\hat{K}_{\chi}(x, s) & =K_{\chi}(x, s), \quad x, s \in \Omega_{N}
\end{aligned}
$$


holds. Under condition (2), let $P_{*, \chi}$ be defined in the same way as $P_{*, \kappa}$. Because of $\chi \in P_{*, \chi}, P_{*, \chi} \neq \varnothing$ holds. Hence, one has

Theorem 3.3.1. Assume the conditions 2.2(3) and (2), and let $\sigma$ be any function from $P_{*, \chi}$. Then the kernel $K_{\sigma}(\cdot, \cdot) \in C\left(\Omega_{N} \times \Omega_{N}\right)$ has an extension $\hat{K}_{\sigma}(\cdot, \cdot) \in C(\Omega \times \Omega)$, i.e.,

$$
\begin{aligned}
\hat{K}_{\sigma}(\cdot, \cdot) & \in C(\Omega \times \Omega) \\
\hat{K}_{\sigma}(x, s) & =K_{\sigma}(x, s), \quad x, s \in \Omega_{N} .
\end{aligned}
$$

Moreover, the following theorem holds.

Theorem 3.3.2. Let the conditions $2.2(3)$ and (2) be satisfied. Then

$$
L_{\sigma}(\Omega)=L_{\chi}(\Omega), \quad \sigma \in P_{*, \chi}
$$

and

$$
\begin{gathered}
\gamma_{0}\|u\|_{1, \sigma} \leq\|u\|_{1, \chi} \leq \gamma_{1}\|u\|_{1, \sigma} \\
u \in L_{\sigma}(\Omega)=L_{\chi}(\Omega)
\end{gathered}
$$

with

$$
\begin{aligned}
& \gamma_{0}=\frac{1}{\sup _{s \in \Omega} \frac{\sigma(s)}{\chi(s)}}=\frac{1}{\left\|\hat{q}_{\sigma}\right\|_{\infty}}, \\
& \gamma_{1}=\sup _{s \in \Omega_{N}} \frac{\chi(s)}{\sigma(s)}=\left\|\hat{q}_{\sigma^{-1}}\right\|_{\infty} .
\end{aligned}
$$

Thus, $L_{\sigma}(\Omega)$ and $L_{\chi}(\Omega)$ are equal as vector spaces, and the associated norms are equivalent.

3.4. Estimates of the eigenvalues and inclusion of the spectral radius for $L_{\sigma}(\Omega)$. In this subsection the proofs are merely outline or even omitted, because they are similar to those of 2.4. First, one has 
Theorem 3.4.1. Let the conditions $2.2(3)$ and $3.2(3)$ be satisfied; further, let $\nu_{\sigma}>0$ be any number and

$$
\int_{\Omega}\left|\hat{K}_{\sigma}(x, s)\right| d x \leq \nu_{\sigma}, \quad s \in \Omega
$$

as well as

$$
\int_{\Omega}\left|\hat{K}_{\sigma}\left(x, s_{0}\right)\right| d x<\nu_{\sigma} \quad \text { for at least one } s_{0} \in \Omega .
$$

Then

$$
|\lambda|<\nu_{\sigma} \quad \text { for all } \lambda \in \Sigma_{1, \sigma}:=\Sigma\left(B: L_{\sigma}(\Omega) \hookrightarrow L_{\sigma}(\Omega)\right) .
$$

Remark 1. Here, as well, the condition (2) is satisfied for all $s_{0} \in N_{K} \subset \partial \Omega$ if $N_{K} \neq \varnothing$ and $\sigma \equiv 1$.

Proof of Theorem 1. (i) $\sigma \equiv 1$. The proof is similar to that of the corresponding part of Theorem 1, Section 2.4; one has to use, in addition, that $\Sigma_{1}^{\prime}:=\Sigma^{\prime}(B: L(\Omega) \hookrightarrow L(\Omega))=\left(\Sigma_{\infty}^{T}\right)^{\prime}:=\Sigma^{\prime}\left(B^{T}:\right.$ $C(\Omega) \hookrightarrow C(\Omega))$.

(ii) General case. Let

$$
\left(\hat{B}_{\sigma} u\right)(x):=\int_{\Omega} \hat{K}_{\sigma}(x, s) u(s) d s, \quad x \in \Omega,
$$

$u \in L(\Omega)$. Then one uses additionally the relation

$$
\begin{aligned}
\Sigma_{1, \sigma}^{\prime} & =\Sigma^{\prime}\left(\hat{B}_{\sigma}: L(\Omega) \hookrightarrow L(\Omega)\right) \\
& =\Sigma^{\prime}\left(\left(\hat{B}_{\sigma}\right)^{T}: C(\Omega) \hookrightarrow C(\Omega)\right) .
\end{aligned}
$$

For the proof of the following Theorem 2, one needs

Lemma 1. Let the condition 2.2(3) be fulfilled, and let additionally $K(x, s) \geq 0, x, s \in \Omega$, be satisfied. Then one has the alternative: either

$$
\int_{\Omega} K(s, x) d s=\rho_{1}(B)\left(=\rho_{\infty}\left(B^{T}\right)=\rho_{1}\left(B^{T}\right)=\rho_{\infty}(B)\right), \quad x \in \Omega
$$


holds or

$$
\min _{x \in \Omega} \int_{\Omega} K(s, x) d s<\rho_{1}(B)<\max _{x \in \Omega} \int_{\Omega} K(s, x) d s .
$$

Theorem 3.4.2. Assume 3.2(2) and $K(x, s) \geq 0, x, s \in \Omega$. Additionally let 2.2(3) be satisfied. Then, for all $\sigma \in P_{*, \chi}$ either

$$
\int_{\Omega} \hat{K}_{\sigma}(s, x) d s=\rho_{1}(B), \quad x \in \Omega
$$

or

$$
\min _{x \in \Omega} \int_{\Omega} \hat{K}_{\sigma}(s, x) d s<\rho_{1}(B)<\max _{x \in \Omega} \int_{\Omega} \hat{K}_{\sigma}(s, x) d s
$$

Moreover, one has the relations

(10) $\sup _{\sigma \in P_{*}, \chi} \min _{x \in \Omega} \int_{\Omega} \hat{K}_{\sigma}(s, x) d s=\rho_{1}(B)=\inf _{\sigma \in P_{*, \chi}} \max _{x \in \Omega} \int_{\Omega} \hat{K}_{\sigma}(s, x) d s$.

Proof. Let

$$
\left(\hat{B}_{\sigma} u\right)(x):=\int_{\Omega} \hat{K}_{\sigma}(x, s) u(s) d s, \quad x \in \Omega,
$$

$u \in C(\Omega)$. Then Lemma 1 implies that either

$$
\int_{\Omega} \hat{K}_{\sigma}(s, x) d s=\rho_{1}\left(\hat{B}_{\sigma}\right), \quad x \in \Omega
$$

or

$$
\min _{x \in \Omega} \int_{\Omega} \hat{K}_{\sigma}(s, x) d s<\rho_{1}\left(\hat{B}_{\sigma}\right)<\max _{x \in \Omega} \int_{\Omega} \hat{K}_{\sigma}(s, x) d s
$$

Now

$$
\int_{\Omega} K(s, x) \chi(s) d s=\rho_{1}(B) \chi(x), \quad x \in \Omega
$$


and, hence,

$$
\rho_{1}(B)=\int_{\Omega} \hat{K}_{\chi}(s, x) d s, \quad x \in \Omega .
$$

This entails

$$
\rho_{1}(B)=\rho_{\infty}\left(\left(\hat{B}_{\chi}\right)^{T}\right)=\rho_{1}\left(\hat{B}_{\chi}\right)=\rho_{1}\left(\hat{B}_{\sigma}\right), \quad \sigma \in P_{*, \chi} .
$$

So the proof is complete.

Finally, it is stated that

$$
\rho_{1, \sigma}(B) \leq \rho_{1, \sigma}(|B|) .
$$

3.5. The convergence criterion in $L_{\sigma}(\Omega)$. As a first application of the estimates for the eigenvalues, one obtains strong and weak convergence criteria as well, which correspond to the strong and weak column-sum criteria for matrices, in this case. In case of the strong criteria, it is again assumed that $K(\cdot, \cdot) \in C(\Omega \times \Omega)$; in case of the weak criterion, condition 2.2(3) is required to be satisfied additionally. Subsequently, this is not mentioned.

(i) Let $N=N_{\sigma}=\varnothing$ and $\sigma \equiv 1$, i.e., $L_{\sigma}(\Omega)=L(\Omega)$ and $\|\cdot\|_{1, \sigma}=\|\cdot\|_{1} ;$ let

$$
q:=\max _{s \in \Omega} \int_{\Omega}|K(x, s)| d x<1 .
$$

Then we say the strong "column-integral criterion" (for short, the strong CIC) is fulfilled. In this case, $\|B\|_{1}=q<1$. For the weak CIC, let

$$
\begin{gathered}
\int_{\Omega}|K(x, s)| d x \leq 1, \quad s \in \Omega \\
\int_{\Omega}\left|K\left(x, s_{0}\right)\right| d x<1 \quad \text { for at least one } s_{0} \in \Omega, \text { if } N_{K}=\varnothing .
\end{gathered}
$$

According to 3.4 , then the estimate $\rho_{1}(|B|)<1$ holds. 
(ii) Let additionally 3.2(3) be satisfied and

$$
q:=\max _{s \in \Omega} \int_{\Omega}\left|\hat{K}_{\sigma}(x, s)\right| d x<1 .
$$

Then we say that the strong $\sigma$-CIC is fulfilled. In this case, $\|B\|_{1, \sigma}=$ $q<1$. For the weak $\sigma$-CIC, let

$$
\begin{gathered}
\int_{\Omega} \mid \hat{K}_{\sigma}(x, s) d x \leq 1, \quad s \in \Omega \\
\int_{\Omega}\left|\hat{K}_{\sigma}\left(x, s_{0}\right)\right| d x<1 \quad \text { for at least one } s_{0} \in \Omega .
\end{gathered}
$$

Then $\rho_{1, \sigma}(|B|)<1$.

(iii) Let additionally condition 3.3(2) be satisfied and

$$
\int_{\Omega}\left|\hat{K}_{\chi}\left(x, s_{0}\right)\right| d x<1 \quad \text { for at least one } s_{0} \in \Omega .
$$

Condition (3) is called $\chi$-CIC; here, the strong and weak criteria are equivalent. Under these conditions,

$$
\rho_{1, \chi}(|B|)=\|B\|_{1, \chi}<1
$$

holds.

4. Examples. This section illustrates the general theorems by examples stemming from boundary value problems of ordinary differential equations. In Example 3, we give a Green's function possessing a logarithmic discontinuity.

Example 1. Let $K(x, s)=G(x, s), x, s \in \Omega=[0, l]$ where

$$
G(x, s)= \begin{cases}s, & 0 \leq s \leq x \leq l \\ x, & 0 \leq x \leq s \leq l\end{cases}
$$

is the Green's function arising in the BVP

$$
-u^{\prime \prime}(x)=\lambda u(x), \quad 0<x<l, \quad u(0)=u^{\prime}(l)=0,
$$




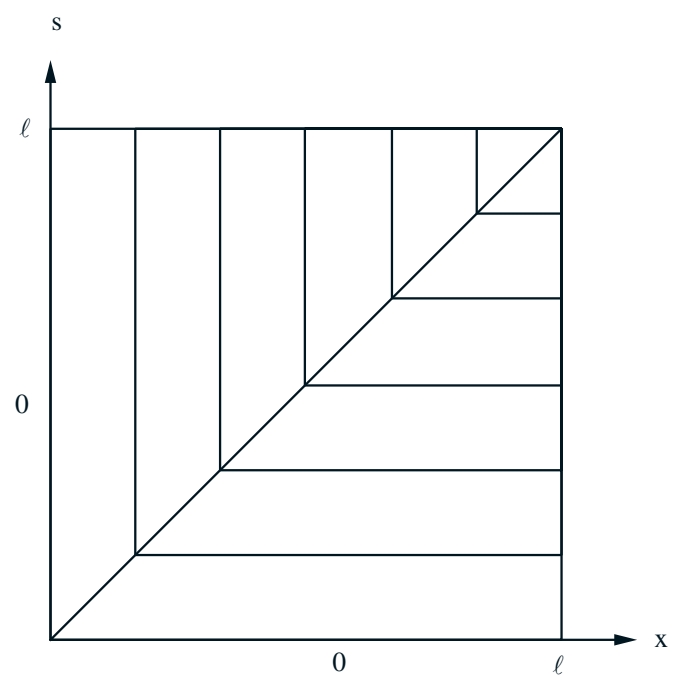

FIGURE 1a. $G(\cdot, \cdot)$ in (1). Illustrated by contour lines.

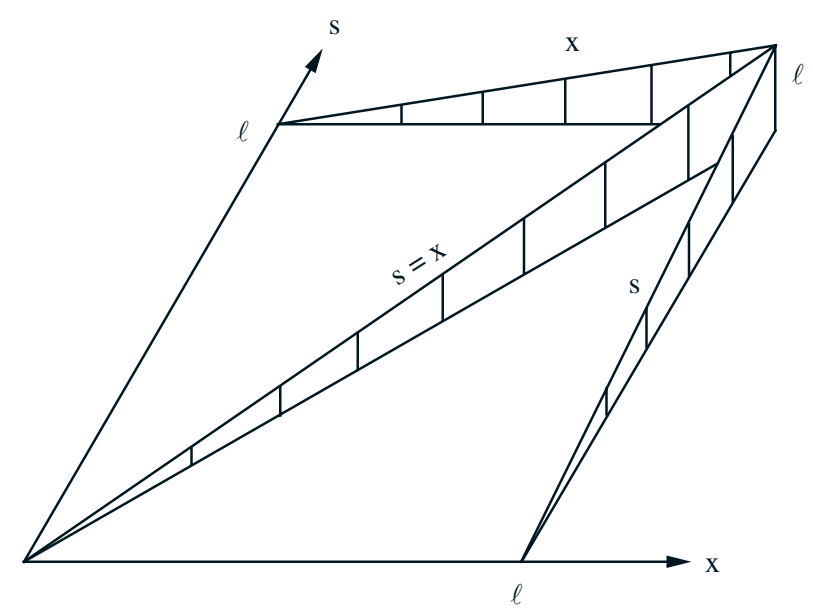

FIGURE 1b. $G(\cdot, \cdot)$ in (1). Illustrated in three dimensions on the boundary and diagonal. 
and which is the eigenvalue problem for a free vibrating string with lefthand end fixed and right-hand end free and horizontal. In this case, $K(\cdot, \cdot)=G(\cdot, \cdot)$ is symmetric. This Green's function is illustrated in Figure 1a by contour lines (cf. [5]) and in Figure 1b in three dimensions on the boundary and on the diagonal. Here $N=N_{K}=\{0\} \subset \partial \Omega=$ $\{0, l\}$.

For $\sigma(x)=(2 x / l)(1-x /(2 l)), x \in \Omega$, the conditions $2.2(6)$ and $3.2(3)$ are fulfilled. Further, here $\kappa(x)=\chi(x)=\sin ((\pi x) /(2 l))$ and the conditions $2.3(2)$ and $3.3(2)$ are satisfied. Moreover, $\sigma \in P_{*, \kappa}$, respectively, $\sigma \in P_{*, \chi}$. The extensions $\hat{K}_{\sigma^{-1}}(\cdot, \cdot)$ and $\hat{K}_{\kappa^{-1}}(\cdot, \cdot)$ of $K_{\sigma^{-1}}(\cdot, \cdot)$ and $K_{\kappa^{-1}}(\cdot, \cdot)$ are given and illustrated qualitatively in Figure 2 on the boundary and on the diagonal. The extensions $\hat{K}_{\sigma}(\cdot, \cdot)$ and $\hat{K}_{\chi}(\cdot, \cdot)$ are the reflected images of $\hat{K}_{\sigma^{-1}}(\cdot, \cdot)$ and $\hat{K}_{\kappa^{-1}}(\cdot, \cdot)$ with respect to the plane orthogonal to the $x-s$-plane and through the diagonal $D$. One has

$$
K_{\sigma^{-1}}(x, s)= \begin{cases}\frac{s^{2}\left(1-\frac{s}{2 l}\right)}{x\left(1-\frac{x}{2 l}\right)}, & 0<s \leq x \leq l \\ \frac{s\left(1-\frac{s}{2 l}\right)}{1-\frac{x}{2 l}}, & 0<x \leq s \leq l\end{cases}
$$

respectively,

$$
K_{\kappa^{-1}}(x, s)= \begin{cases}\frac{s \sin \frac{\pi}{2 l} s}{\sin \frac{\pi}{2 l} x}, & 0<s \leq x \leq l \\ \frac{x \sin \frac{\pi}{2 l} s}{\sin \frac{\pi}{2 l} x}, & 0<x \leq s \leq l\end{cases}
$$

In Theorem 2.3.2, the constants are given by $\gamma_{0}=1$ and $\gamma_{1}=4 / \pi$, and in Theorem 3.3.2, by $\gamma_{0}=\pi / 4$ and $\gamma_{1}=1$.

In the spaces $C(\Omega)$ and $L(\Omega)$, the strong RIC and the strong CIC in Section 6 are fulfilled, if

$$
\frac{l^{2}}{2}<1, \quad \text { i.e., } l<\sqrt{2} \doteq 1.414,
$$

and the weak RIC and the weak CIC are satisfied, if

$$
\frac{l^{2}}{2} \leq 1, \quad \text { i.e., } l \leq \sqrt{2} \doteq 1.414 \text {. }
$$




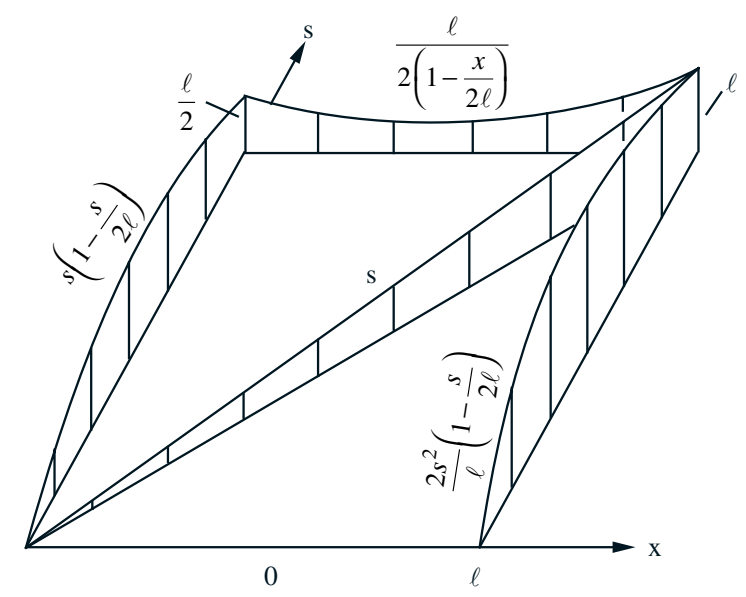

FIGURE 2a. $\hat{K}_{\sigma^{-1}}(\cdot, \cdot)$, respectively $\hat{K}_{\kappa^{-1}}(\cdot, \cdot)$ on $\partial(\Omega \times \Omega)$ and on $D$.

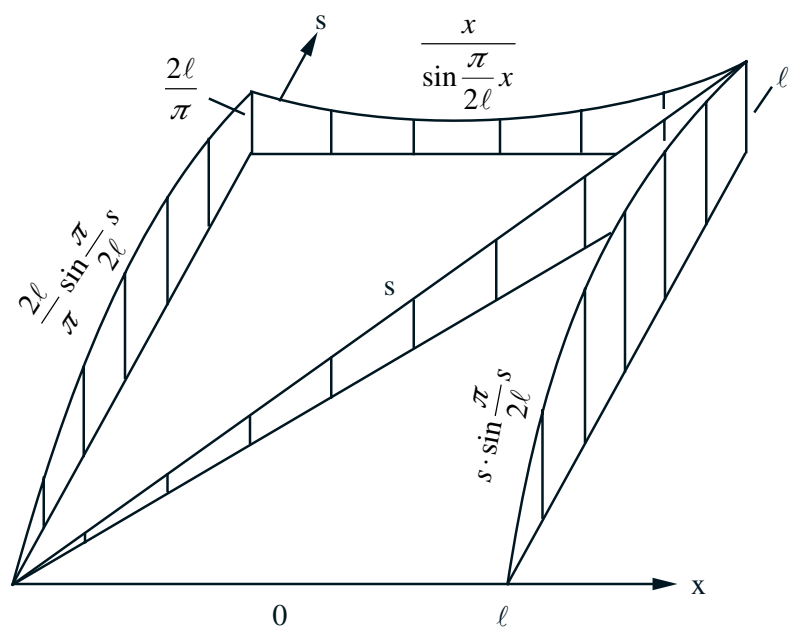

FIGURE 2b. 
In the spaces $C_{\sigma^{-1}}(\Omega)$ and $L_{\sigma}(\Omega)$, the strong $\sigma^{-1}$-RIC and the strong $\sigma$-CIC are satisfied for

$$
\frac{5}{6} \frac{l^{2}}{2}<1, \quad \text { i.e., } l<\sqrt{\frac{6}{5}} \sqrt{2} \doteq 1.549,
$$

and the weak $\sigma^{-1}$-RIC and the weak $\sigma$-CIC when

$$
\frac{5}{6} \frac{l^{2}}{2} \leq 1, \quad \text { i.e., } l \leq \sqrt{\frac{6}{5}} \sqrt{2} \doteq 1.549 .
$$

Finally, in the spaces $C_{\kappa^{-1}}(\Omega)$ and $L_{\chi}(\Omega)$, the $\kappa^{-1}$-RIC and the $\chi$-CIC are satisfied, if

(6) $\rho(B)=\|B\|_{\infty, \kappa^{-1}}=\|B\|_{1, \chi}=\left(\frac{2 l}{\pi}\right)^{2}<1$

$$
\text { respectively } l<\frac{\pi}{2} \doteq 1.571 \text {. }
$$

Further, the relations 2.4(25), respectively 3.4(9), are fulfilled, which are for $\sigma \equiv 1$, respectively $\sigma(x)=(2 l / x)(1-x /(2 l))$ equivalent to

$$
0<\frac{4}{\pi^{2}} l^{2}<\frac{l^{2}}{2}, \quad \text { i.e., } 0<0.405 \cdots l^{2}<0.5 l^{2},
$$

respectively,

(8) $\frac{2}{3} \frac{l^{2}}{2}<\frac{4}{\pi^{2}} l^{2}<\frac{5}{6} \frac{l^{2}}{2}$, i.e., $0.333 \cdots l^{2}<0.405 \cdots l^{2}<0.416 \cdots l^{2}$.

Example 2. We take $K(x, s)=G(x, s), x, s \in \Omega=[0, l]$ where

$$
G(x, s)= \begin{cases}(1-x / l) s, & 0 \leq s \leq x \leq l \\ (1-s / l) x, & 0 \leq x \leq s \leq l\end{cases}
$$

is the Green's function arising in the BVP

$$
-u^{\prime \prime}(x)=\lambda u(x), \quad 0<x<l, \quad u(0)=u(l)=0 .
$$

Hence, the string has fixed ends in this case. The Green's function is illustrated in Figure 3a by contour lines (cf. [5]) and in Figure 3b 


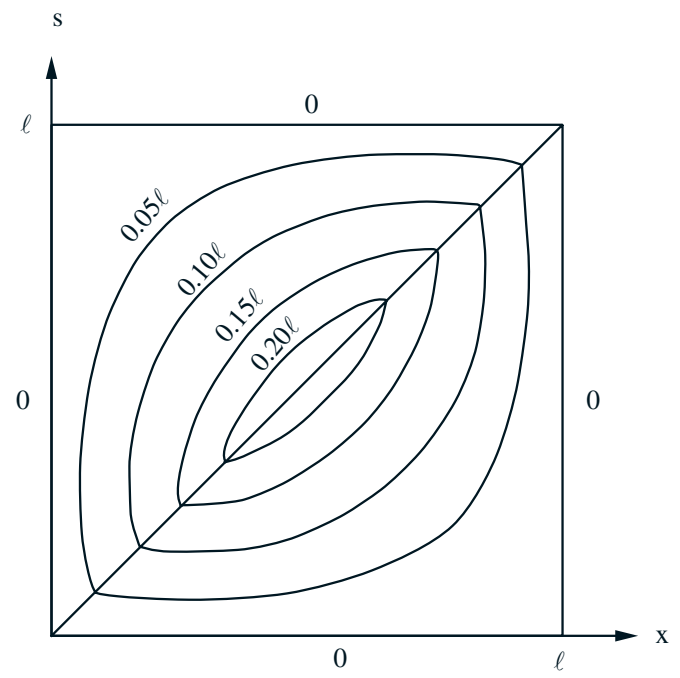

FIGURE 3a. $G(\cdot, \cdot)$ in $(\mathrm{g})$. Illustrated by contour lines.

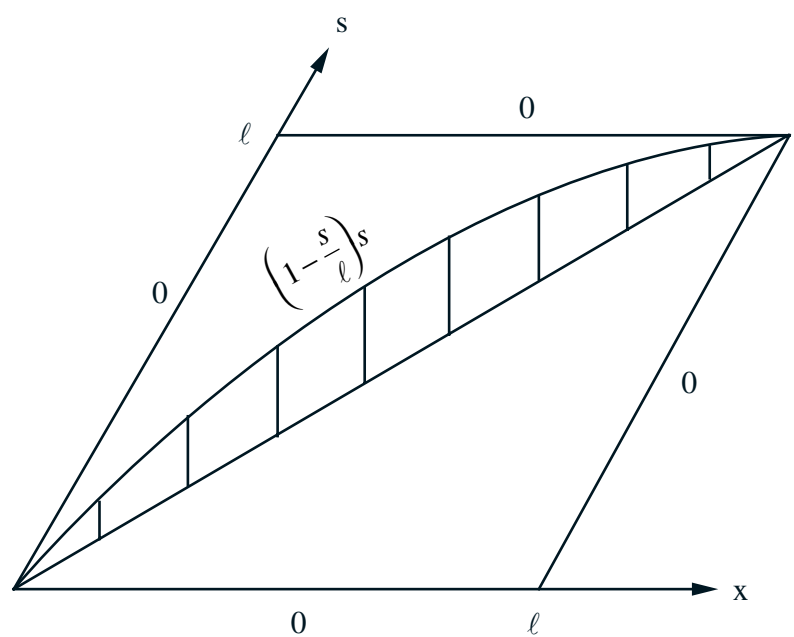

FIGURE 3b. $G(\cdot, \cdot)$ in $(\mathrm{g})$. Illustrated in three dimensions on the boundary and diagonal. 
in three dimensions on the boundary and on the diagonal. Here $N=N_{K}=\{0, l\}=\partial \Omega$.

For $\sigma(x)=(x / l)(1-x / l), x \in \Omega$, the conditions 2.2(6) and 3.2(3) are fulfilled. Further, here $\kappa(x)=\chi(x)=\sin (\pi x / l)$, and the conditions 2.3(2) and 3.3(2) are satisfied. Moreover, $\sigma \in P_{*, \kappa}$, respectively $\sigma \in P_{*, \chi}$. The extensions $\hat{K}_{\sigma^{-1}}(\cdot, \cdot)$ and $\hat{K}_{\kappa^{-1}}(\cdot, \cdot)$ of $K_{\sigma^{-1}}(\cdot, \cdot)$ and $K_{\kappa^{-1}}(\cdot, \cdot)$ are given and illustrated qualitatively in Figure 4 on the boundary and on the diagonal. The extensions $\hat{K}_{\sigma}(\cdot, \cdot)$ and $\hat{K}_{\chi}(\cdot, \cdot)$ are again the reflected images of $\hat{K}_{\sigma^{-1}}(\cdot, \cdot)$ and $\hat{K}_{\kappa^{-1}}(\cdot, \cdot)$ with respect to the plane orthogonal to the $x-s$ plane and through the diagonal $D$. One has

$$
K_{\sigma^{-1}}(x, s)= \begin{cases}\frac{s^{2}(1-s / l)}{x}, & 0<s \leq x<l \\ \frac{s(1-s / l)^{2}}{1-x / l}, & 0<x \leq s<l\end{cases}
$$

and

$$
K_{\kappa^{-1}}(x, s)= \begin{cases}\frac{(1-x / l) s \sin (\pi s / l)}{\sin (\pi x / l)}, & 0<s \leq x<l \\ \frac{(1-s / l) x \sin (\pi s / l)}{\sin (\pi x / l)}, & 0<x \leq s<l\end{cases}
$$

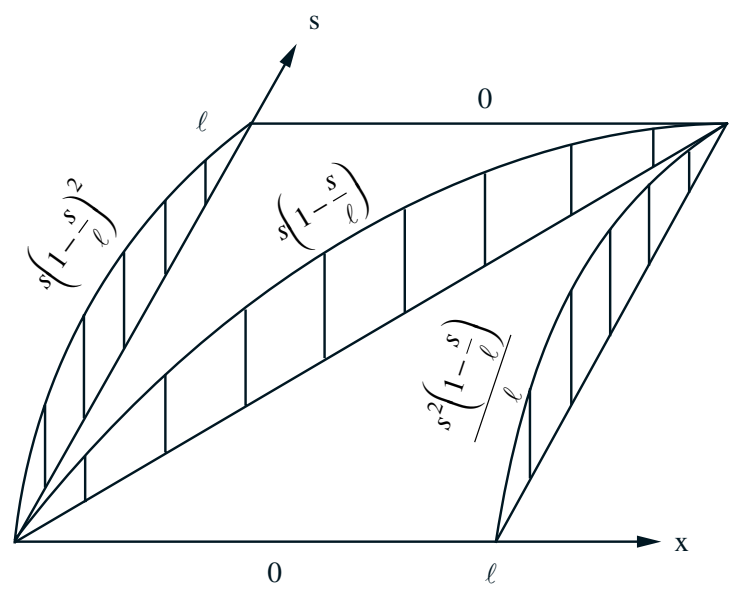

FIGURE 4a. $\hat{K}_{\sigma^{-1}}(\cdot, \cdot)$, respectively $\hat{K}_{\kappa^{-1}}(\cdot, \cdot)$ on $\partial(\Omega \times \Omega)$ and on $D$. 


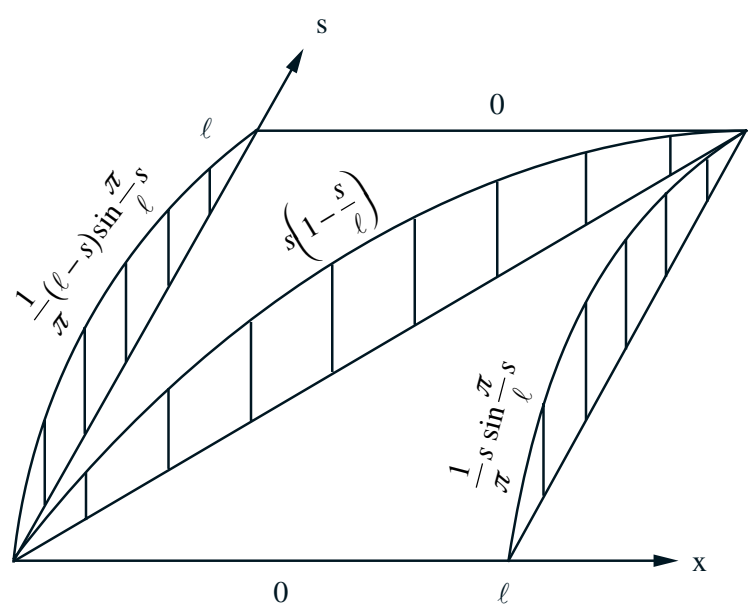

FIGURE $4 b$.

In Theorem 2.3.2, one has $\gamma_{0}=1 / 4$ and $\gamma_{1}=1 / \pi$, and in Theorem 3.3.2 the constants $\gamma_{0}=\pi$ and $\gamma_{1}=4$.

The convergence criteria are omitted here because they are given in a different setting in Section 5 .

Further, the relations 2.4(25), respectively, 3.4(9) are fulfilled, which are for $\sigma \equiv 1$, respectively $\sigma(x)=(x / l)(1-x / l)$ equivalent to

$$
0<\frac{l^{2}}{\pi^{2}}<\frac{l^{2}}{8}, \quad \text { i.e., } 0<0.101 \cdots l^{2}<0.125 l^{2},
$$

respectively,

$$
\frac{2}{3} \frac{l^{2}}{8}<\frac{l^{2}}{\pi^{2}}<\frac{5}{6} \frac{l^{2}}{8}, \quad \text { i.e., } 0.08333 \cdots l^{2}<0.101 \cdots l^{2}<0.104 \cdots l^{2} .
$$

Example 3. So far, the kernel $K(\cdot, \cdot)$ was supposed to be continuous on $\Omega \times \Omega$. But the Green's function corresponding to an ordinary differential operator can also have a logarithmic discontinuity. For example, the Green's function for the differential expression

$$
x u^{\prime \prime}(x)+u^{\prime}(x), \quad 0<x<1,
$$


and the boundary conditions

$$
u(1)=0, \quad u(0) \text { finite }
$$

is given by

$$
K(x, s)=G(x, s)= \begin{cases}-\ln x, & 0<s \leq x \leq 1 \\ -\ln s, & 0<x \leq s \leq 1\end{cases}
$$

the differential expression (14) with conditions (15) is associated with the Bessel's function $\mathcal{T}_{0}(\kappa)$ of order zero (cf. [6, p. 321]). Here $N=\{1\} \subset \partial \Omega=\{0,1\}$ and instead of 2.2(6), respectively 3.2(3), one must assume, e.g., that the limit

$$
\lim _{\substack{\tilde{x} \rightarrow x \\ \tilde{x} \in \Omega_{N}}} \int_{\Omega} K_{\sigma^{-1}}(\tilde{x}, s) d s, \quad x \in N,
$$

respectively

$$
\lim _{\substack{\tilde{s} \rightarrow s \\ \tilde{s} \in \Omega_{N}}} \int_{\Omega} K_{\sigma}(x, \tilde{s}) d s, \quad s \in N
$$

exists. Setting, for instance, $\sigma(x)=1-x, x \in \Omega=[0,1]$, then

$$
\int_{\Omega} K_{\sigma^{-1}}(x, s) d s=1-\frac{1+x}{4}, \quad x \in[0,1),
$$

and hence

$$
\lim _{\substack{\tilde{x} \rightarrow 1 \\ \tilde{x} \in[0,1)}} \int_{0}^{1} K_{\sigma^{-1}}(x, 1) d s=\frac{1}{2} .
$$

So, with these alternations, such problems can also be handled similar to the case $K(\cdot, \cdot) \in C(\Omega \times \Omega)$. The details are omitted for the sake of brevity.

5. Application to a boundary value problem. In this section we consider a boundary value problem of an ordinary differential equation in physics, respectively engineering, its reformulation as an integral 
equation as well as convergence criteria in unweighted and weighted norms. Especially, the convergence criteria of Section 4 are applicable to the example. The problem is solved by the Jacobi method for specific data. Numerical tests confirm the theoretical results.

5.1. Elastically supported string. From [4], the elastically supported string is described by the BVP

$$
\begin{gathered}
-u^{\prime \prime}(x)+c u(x)=f(x), \quad 0<x<l \\
u(0)=u(l)=0 .
\end{gathered}
$$

Here $c>0$ describes the elastic support, $u$ means the vertical displacement, and $f$ the load per unit length. As for $f$, we assume, e.g., that $f \in C[0, l]$. In this case the solution $u \in C_{0}(\Omega) \cap C^{2}(\Omega), \Omega:=[0, l]$, with $C_{0}(\Omega):=\{u \in C(\Omega) \mid u(0)=u(l)=0\}$ being searched for. Or, let $f \in L_{2}(\Omega)$ and consider the above BVP in the sense of distributions, i.e.,

$$
\begin{gathered}
\int_{0}^{l} u^{\prime}(x) \varphi^{\prime}(x) d x+c \int_{0}^{l} u(x) \varphi(x) d x=\int_{0}^{l} f(x) \varphi(x) d x, \\
\varphi \in C_{0}^{\infty}(\stackrel{\circ}{\Omega}) .
\end{gathered}
$$

Then a solution $u \in H_{0}^{1}(\stackrel{\circ}{\Omega}) \cap H^{2}(\stackrel{\circ}{\Omega})$ is sought. Particularly, in this case $u \in C_{1}(\Omega)$ holds because of Sobolev's inequality. This will be confirmed in another way by the integral representation of the solution.

In both cases, the solution can be given by the Green's function of the differential operator $-u^{\prime \prime}(x)+c u(x)$ :

$$
u(x)=\int_{\Omega} \tilde{G}(x, s, c) f(s) d s, \quad x \in \Omega
$$

with

$$
\tilde{G}(x, s, c)= \begin{cases}\frac{\sinh \sqrt{c}(l-x)}{\sinh (\sqrt{c} l)} \frac{\sinh (\sqrt{c} s)}{\sqrt{c}}, & 0 \leq s \leq x \leq l \\ \frac{\sinh \sqrt{c}(l-s)}{\sinh (\sqrt{c} l)} \frac{\sinh (\sqrt{c} x)}{\sqrt{c}}, & 0 \leq x \leq s \leq l .\end{cases}
$$


Here also $\tilde{G}(x, s, c) \geq 0, x, s \in \Omega$.

So BVP (1), (2) is exactly solvable and can serve as a test problem. To solve (1) and (2) by the Jacobi method, let $G(x, s)$ be the Green's function of $-u^{\prime \prime}$. Then one has

$$
u(x)=-c \int_{\Omega} G(x, s) u(s) d s+g(x), \quad x \in \Omega
$$

with

$$
g(x)=\int_{\Omega} G(x, s) f(s) d s, \quad x \in \Omega,
$$

i.e.,

$$
u(x)=\int_{\Omega} K(x, s) u(s) d s+g(x), \quad x \in \Omega
$$

with

$$
K(x, s)=-c G(x, s)=: K(x, s, c), \quad x, s \in \Omega,
$$

where $G(\cdot, \cdot)$ is given by

$$
G(x, s)= \begin{cases}(1-x / l) s, & 0 \leq s \leq x \leq l \\ (1-s / l) x, & 0 \leq x \leq s \leq l .\end{cases}
$$

Here there holds

$$
K(x, s) \leq 0, \quad x, s \in \Omega,
$$

whence

$$
|K(x, s)|=-K(x, s)=c G(x, s), \quad x, s \in \Omega .
$$

Remark. When the above differential equation (1) is studied under the boundary conditions $u(0)=u^{\prime}(l)=0$, then one has

$$
G(x, s)= \begin{cases}s, & 0 \leq s \leq x \leq l \\ x, & 0 \leq x \leq s \leq l .\end{cases}
$$


We shall not pursue this case further.

5.2. The convergence criteria. In the spaces $C(\Omega)$ and $L(\Omega)$, the strong RIC and the strong CIC are satisfied, if

$$
c \frac{l^{2}}{8}<1
$$

and the weak RIC and the weak CIC, if

$$
c \frac{l^{2}}{8} \leq 1
$$

In the spaces $C_{\sigma^{-1}}(\Omega)$ and $L_{\sigma}(\Omega)$ with $\sigma(x)=(x / l)(1-x / l), x \in \Omega$, the strong $\sigma^{-1}$-RIC and the strong $\sigma$-CIC are satisfied for

$$
c \frac{5}{6} \frac{l^{2}}{8}<1
$$

and the weak $\sigma^{-1}$-RIC and weak $\sigma$-CIC, when

$$
c \frac{5}{6} \frac{l^{2}}{8} \leq 1
$$

Finally, in the spaces $C_{\kappa^{-1}}(\Omega)$ and $L_{\chi}(\Omega)$ with $\kappa(x)=\chi(x)=$ $\sin (\pi x / l), x \in \Omega$, the $\kappa^{-1}$-RIC and the $\chi$-CIC are satisfied, if

$$
c \frac{l^{2}}{\pi^{2}}<1 .
$$

\subsection{Numerical example.}

(i) Problem and exact solution. As a numerical example, we consider the BVP

$$
\begin{gathered}
-u^{\prime \prime}+u=1, \quad x \in(0,1) \\
u(0)=u(1)=0,
\end{gathered}
$$

i.e., $c=1, l=1, f(x) \equiv 1$. The exact solution is given by

$$
z(x)=u_{\text {exact }}(x)=\int_{0}^{1} \tilde{G}(x, s, c=1) d s
$$


with

$$
\tilde{G}(x, s, c=1)= \begin{cases}\frac{\sinh (1-x)}{\sinh (1)} \sinh s, & 0 \leq s \leq x \leq 1 \\ \frac{\sinh (1-s)}{\sinh (1)} \sinh x, & 0 \leq x \leq s \leq 1 .\end{cases}
$$

From (3) and (4), one infers

$$
\begin{aligned}
z(x) & =u_{\text {exact }}(x) \\
& =\frac{1}{\sinh (1)}\{\sinh (1-x)[\cosh (x)-1]+\sinh (x)[\cosh (1-x)-1]\}
\end{aligned}
$$

(ii) The Jacobi method. Let $G(\cdot, \cdot)$ be the Green's function corresponding to $-u^{\prime \prime}$. Then

$$
u(x)=\int_{0}^{1} K(x, s) u(s) d s+g(x), \quad x \in \Omega=[0,1]
$$

with

$$
\begin{aligned}
G(x, s) & = \begin{cases}(1-x) s, & 0 \leq s \leq x \leq 1 \\
(1-s) x, & 0 \leq x \leq s \leq 1,\end{cases} \\
g(x) & =\int_{0}^{1} G(x, s) d s=\frac{x(1-x)}{2}, \quad x \in[0,1],
\end{aligned}
$$

and

$$
K(x, s)=-G(x, s), \quad x, s \in[0,1] .
$$

The spectral radius of

$$
|B| u(x):=\int_{0}^{1}|K(x, s)| u(s) d s=\int_{0}^{1} G(x, s) u(s) d s, \quad 0 \leq x \leq 1,
$$

is given by

$$
\rho(|B|)=\rho_{\infty}(|B|)=\rho(-B)=\frac{1}{\pi^{2}}<1
$$


and the corresponding eigenfunction reads

$$
\kappa(x)=\sin \pi x, \quad 0 \leq x \leq 1
$$

The Jacobi method can be considered, e.g., in the spaces $V=$ $C_{\kappa^{-1}}[0,1]$, respectively, $V=C[0,1]$. It is given by

$$
u_{t+1}(x)=\int_{0}^{1} K(x, s) u_{t}(1) d s+g(x), \quad 0 \leq x \leq 1
$$

$t=0,1,2, \ldots$, with any $u_{0} \in V$.

(iii) Error estimates and numerical results. In $V=C_{\kappa^{-1}}[0,1]$, the error estimate reads

$$
\begin{aligned}
\left\|u_{t}-z\right\|_{\infty, \kappa^{-1}} & \leq \frac{\rho_{\infty}(|B|)}{1-\rho_{\infty}(|B|)}\left\|u_{t}-u_{t-1}\right\|_{\infty, \kappa^{-1}} \\
& \leq \frac{\rho_{\infty}^{t}(|B|)}{1-\rho_{\infty}(|B|)}\left\|u_{1}-u_{0}\right\|_{\infty, \kappa^{-1}} \rightarrow 0, \quad t \rightarrow \infty
\end{aligned}
$$

$t=1,2, \ldots$ and correspondingly in the space $V=C[0,1]$,

$$
\begin{aligned}
\left\|u_{t}-z\right\|_{\infty} & \leq \frac{\|B\|_{\infty}}{1-\|B\|_{\infty}}\left\|u_{t}-u_{t-1}\right\|_{\infty} \\
& \leq \frac{\|B\|_{\infty}^{t}}{1-\|B\|_{\infty}}\left\|u_{1}-u_{0}\right\|_{\infty} \rightarrow 0, \quad t \rightarrow \infty
\end{aligned}
$$

with $u_{0} \in V$, respectively. Fully written, this means

$$
\begin{aligned}
\sup _{x \in(0,1)} \frac{\left|u_{t}(x)-z(x)\right|}{\sin \pi x} & \leq \frac{\frac{1}{\pi^{2}}}{1-\frac{1}{\pi^{2}}} \sup _{x \in(0,1)} \frac{\mid u_{t}(x)-u_{t-1}(x)}{\sin \pi x} \\
& \leq \frac{\left(\frac{1}{\pi^{2}}\right)^{t}}{1-\frac{1}{\pi^{2}}} \sup _{x \in(0,1)} \frac{\left|u_{1}(x)-u_{0}(x)\right|}{\sin \pi x} \rightarrow 0, \quad t \rightarrow \infty
\end{aligned}
$$

respectively,

$$
\begin{aligned}
\max _{x \in[0,1]}\left|u_{t}(x)-z(x)\right| & \leq \frac{\frac{1}{8}}{1-\frac{1}{8}} \max _{x \in[0,1]}\left|u_{t}(x)-u_{t-1}(x)\right| \\
& \leq \frac{\left(\frac{1}{8}\right)^{t}}{1-\frac{1}{8}} \max _{x \in[0,1]}\left|u_{1}(x)-u_{0}(x)\right| \rightarrow 0, \quad t \rightarrow \infty
\end{aligned}
$$


For $u_{0}(x) \equiv 0 \in V=C_{\kappa^{-1}}[0,1]$, respectively $V=C[0,1]$,

$$
\begin{aligned}
u_{0}(x) & \equiv 0 \\
u_{1}(x) & =\frac{x(1-x)}{2} \\
u_{2}(x) & =\frac{x(1-x)}{24}\left(x^{2}-x+11\right)=\frac{x(1-x)}{2} \frac{11-x(1-x)}{12} \\
u_{3}(x) & =\frac{x(1-x)}{24 \cdot 60}\left[2 x^{4}-4 x^{3}+56 x^{2}-54 x+666\right] \\
& =\frac{x(1-x)}{24 \cdot 60}\{666+2 \times(1-x)[x(1-x)-27]\} \\
\vdots & \quad+\sinh (x)[\cosh (1-x)-1]\} .
\end{aligned}
$$

holds. Further, one has

$$
\begin{aligned}
\sup _{x \in(0,1)} \frac{\left|u_{t}(x)-z(x)\right|}{\sin \pi x} & =\lim _{x \rightarrow 0} \frac{\left|u_{t}(x)-z(x)\right|}{\sin \pi x} \\
& =\lim _{x \rightarrow 1} \frac{\left|u_{t}(x)-z(x)\right|}{\sin \pi x}, \\
\sup _{x \in(0,1)} \frac{\left|u_{t}(x)-u_{t-1}(x)\right|}{\sin \pi x} & =\lim _{x \rightarrow 0} \frac{\left|u_{t}(x)-u_{t-1}(x)\right|}{\sin \pi x} \\
& =\lim _{x \rightarrow 1} \frac{\left|u_{t}(x)-u_{t-1}(x)\right|}{\sin \pi x}
\end{aligned}
$$

$t=1,2, \ldots$, as well as

$$
\begin{aligned}
\max _{x \in[0,1]}\left|u_{t}(x)-z(x)\right| & =\left|u_{t}\left(\frac{1}{2}\right)-z\left(\frac{1}{2}\right)\right| \\
\max _{x \in[0,1]}\left|u_{t}(x)-u_{t-1}(x)\right| & =\left|u_{t}\left(\frac{1}{2}\right)-u_{t-1}\left(\frac{1}{2}\right)\right|, \quad t=1,2, \ldots .
\end{aligned}
$$

The error estimates in $V=C_{\kappa^{-1}}[0,1]$, respectively $V=C[0,1]$, along with the numerical values are compiled in Tables 1 and 2 . 
TABLE 1. Error estimates in the norm $\|\cdot\|_{\infty, \kappa^{-1}}$ for $t=1,2,3$.

\begin{tabular}{|l|}
\hline$\left\|u_{1}-z\right\|_{\infty} \leq\left\|u_{1}-z\right\|_{\infty, \kappa^{-1}} \leq \frac{\frac{1}{\pi^{2}}}{1-\frac{1}{\pi^{2}}}\left\|u_{1}-u_{0}\right\|_{\infty, \kappa^{-1}}$ \\
$0.01181889 \leq 0.01205848 \leq 0.017944373$ \\
\hline$\left\|u_{2}-z\right\|_{\infty} \leq\left\|u_{2}-z\right\|_{\infty, \kappa^{-1}} \leq \frac{\frac{1}{\pi^{2}}}{1-\frac{1}{\pi^{2}}}\left\|u_{2}-u_{1}\right\|_{\infty, \kappa^{-1}}$ \\
$\leq \frac{\left(\frac{1}{\pi^{2}}\right)^{2}}{1-\frac{1}{\pi^{2}}}\left\|u_{1}-u_{0}\right\|_{\infty, \kappa^{-1}}$ \\
$0.00120194 \leq 0.00120443 \leq 0.00149532 \leq 0.00181809$ \\
$\left\|u_{3}-z\right\|_{\infty} \leq\left\|u_{3}-z\right\|_{\infty, \kappa^{-1}} \leq \frac{\frac{1}{\pi^{2}}}{1-\frac{1}{\pi^{2}}}\left\|u_{3}-u_{2}\right\|_{\infty, \kappa^{-1}}$ \\
$\leq \frac{\left(\frac{1}{\pi^{2}}\right)^{2}}{1-\frac{1}{\pi^{2}}}\left\|u_{2}-u_{1}\right\|_{\infty, \kappa^{-1}} \leq \frac{\left(\frac{1}{\pi^{2}}\right)^{3}}{1-\frac{1}{\pi^{2}}}\left\|u_{1}-u_{0}\right\|_{\infty, \kappa^{-1}}$ \\
$0.00012184 \leq 0.00012186 \leq 0.00014853$ \\
$\leq 0.00015151 \leq 0.00018421$
\end{tabular}

TABLE 2. Error estimates in the norm $\|\cdot\|_{\infty}$ for $t=1,2,3$.

\begin{tabular}{|l|}
$\left\|u_{1}-z\right\|_{\infty} \leq \frac{\frac{1}{8}}{1-\frac{1}{8}}\left\|u_{1}-u_{0}\right\|_{\infty}$ \\
$0.01181889 \leq 0.01785714$ \\
\hline$\left\|u_{2}-z\right\|_{\infty} \leq \frac{\frac{1}{8}}{1-\frac{1}{8}}\left\|u_{2}-u_{1}\right\|_{\infty} \leq \frac{\left(\frac{1}{8}\right)^{2}}{1-\frac{1}{8}}\left\|u_{1}-u_{0}\right\|_{\infty}$ \\
$0.00120194 \leq 0.00186012 \leq 0.00223214$ \\
$\left\|u_{3}-z\right\|_{\infty} \leq \frac{\frac{1}{8}}{1-\frac{1}{8}}\left\|u_{3}-u_{2}\right\|_{\infty}$ \\
$\leq \frac{\left(\frac{1}{8}\right)^{2}}{1-\frac{1}{8}}\left\|u_{2}-u_{1}\right\|_{\infty} \leq \frac{\left(\frac{1}{8}\right)^{3}}{1-\frac{1}{8}}\left\|u_{1}-u_{0}\right\|_{\infty}$ \\
$0.00012184 \leq 0.00018911 \leq 0.00023265 \leq 0.00027902$
\end{tabular}




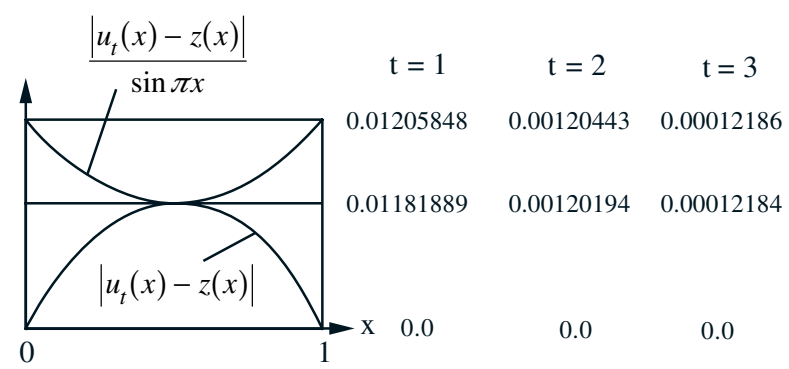

FIGURE 5. Qualitative course of the graphs of $\left|u_{t}(x)-z(x)\right| / \sin \pi x$ and of $\left|u_{t}(x)-z(x)\right|$ as well as corresponding ordinate ranges.

Because of

$$
\max _{x \in[0,1]}\left|u_{t}(x)-z(x)\right| \leq \sup _{x \in(0,1)} \frac{\left|u_{t}(x)-z(x)\right|}{\sin \pi x}, \quad t=0,1,2, \ldots,
$$

one also obtains with the estimates in $C_{\kappa^{-1}}[0,1]$ estimates in the space $C[0,1]$.

Looking at the tables, it can be seen that the estimates in $C_{\kappa^{-1}}[0,1]$ begin at a higher level, which can also be deduced from (19). The a posteriori and a priori error estimates are then better than in $C[0,1]$, however. This becomes especially clear by comparing the curves of $\left|u_{t}(x)-z(x)\right| / \sin \pi x, x \in(0,1)$, and $\left|u_{t}(x)-z(x)\right|, x \in[0,1]$, in the same coordinate system. The qualitative course of these curves can be seen in Figure 5. The ordinate range for $\left|u_{t}(x)-z(x)\right| / \sin \pi x$ is drawn outsized. The ratio of the ordinate ranges is compiled in Table 3 . On the whole, the results show that, with weighted norms, the error estimates can be sharpened.

TABLE 3. Ratio of the ordinate ranges according to Figure 5.

\begin{tabular}{|l|l|}
\hline$t$ & $\frac{\sup _{x \in(0,1)} \frac{\left|u_{t}(x)-z(x)\right|}{\sin \pi x}-\inf _{x \in(0,1)} \frac{\left|u_{t}(x)-z(x)\right|}{\sin \pi x}}{\max _{x \in[0,1]}\left|u_{t}(x)-z(x)\right|-\min _{x \in[0,1]}\left|u_{t}(x)-z(x)\right|}$ \\
\hline 1 & $\frac{0.01205848-0.01181889}{0.01181899-0.0} \doteq 0.027$ \\
\hline 2 & $\frac{0.00120443-0.00120194}{0.00120194-0.0} \doteq 0.0021$ \\
\hline 3 & $\frac{0.00012186-0.00012184}{0.00012184-0.0} \doteq 0.00016$ \\
\hline
\end{tabular}


Acknowledgment. The author thanks the referees for their valuable comments which led to a better presentation of the paper. Thanks also to Prof. P.M. Anselone for helping to polish the English.

\section{REFERENCES}

1. R. Adams, Sobolev spaces, Academic Press, New York, 1975.

2. G. Bachman and L. Narici, Functional analysis, Academic Press, New York, 1966.

3. L. Collatz, Über die Konvergenzkriterien bei Iterationsverfahren für lineare Gleichungssysteme, Math. Z. 53 (1950), 149-161.

4. - Eigenwertprobleme mit technischen Anwendungen, Akademische Verlagsgesellschaft, Leipzig, 1963.

5. — Differentialgleichungen, Teubner, Stuttgart, 1973.

6. R. Courant and D. Hilbert, Methoden der mathematischen, Physik I, Springer Verlag, Berlin, 1968.

7. R. Jentzsch, Über Integralgleichungen mit positivem Kern., J. Reine Angew. Math. 141 (1912), 235-244.

8. L.W. Kantorowitsch and G.P. Akilow, Funktionalanalysis in normierten Räumen, Akademie-Verlag, Berlin, 1964.

9. S. Karlin, Positive operators, J. Math. Mech. 8 (1959), 907-937.

10. M.A. Krasnosel'skij, Je.A. Lifshits and A.V. Sobolev, Positive linear systems — The method of positive operators, Heldermann Verlag, Berlin, 1989.

11. M.G. Krein and M.A. Rutman, Linear operators leaving invariant a cone of a Banach space (Russian), Uspechi mate. nauk 3 (23) 1948, 3-95; English transl. in Amer. Math. Soc. Transl., vol. 26.

12. N. Luther, K. Niederdrenk, F. Reutter and H. Yserentant, Gewöhnliche Differentialgleichungen, Friedr. Vieweg \& Sohn, Braunschweig Wiesbaden, 1987.

13. I.P. Natanson, Theorie der Funktionen einer reellen Veränderlichen, Akademie-Verlag, Berlin, 1961.

14. J.T. Oden, Applied functional analysis, Prentice-Hall, Englewood Cliffs, NJ, 1979.

15. A.E. Taylor, Introduction to functional analysis, John Wiley \& Sons, London, 1958.

16. J. Weissinger, Zur Theorie und Anwendung des Iterationsverfahrens, Math. Nachr. 8 (1952), 193-212.

Prager Str. 9, D-10779 Berlin 